\title{
Stability of Bifurcating Patterns of Spatial Economy Models on a Hexagonal Lattice
}

\author{
K. Ikeda*, H. Aizawa ${ }^{\dagger}$ and Y. Kogure ${ }^{\ddagger}$ \\ Department of Civil and Environmental Engineering, \\ Tohoku University, Aoba, Sendai 980-8579, Japan \\ *kiyohiro.ikeda.b4@tohoku.ac.jp \\ thiroki.aizawa.p3@dc.tohoku.ac.jp \\ ${ }^{\ddagger}$ yosuke.kogure.t2@dc.tohoku.ac.jp \\ Y. Takayama \\ Institute of Science and Engineering, \\ Kanazawa University, Kakuma, \\ Kanazawa 920-1192, Japan \\ ytakayama@se.kanazawa-u.ac.jp
}

Received March 22, 2018; Revised June 17, 2018

\begin{abstract}
Self-organization of spatial patterns is investigated for a scalar field of a system of locations on a hexagonal lattice. Group-theoretic bifurcation analysis is conducted to exhaustively try and find possible bifurcating patterns. All these patterns are proved to be asymptotically unstable for general spatial economic models in new economic geography. Microeconomic interactions among the locations are expressed by a spatial economy model and all bifurcating patterns are demonstrated to be unstable by numerical bifurcation analysis.
\end{abstract}

Keywords: Group-theoretic bifurcation theory; hexagonal lattice; self-organization; spatial economy; stability.

\section{Introduction}

The mechanism of self-organization of hexagonal distributions of cities was envisaged in central place theory of economic geography (Fig. 1 1 of Christaller, 1933]), and has come untangled by group-theoretic bifurcation theory Ikeda \& Murota, 2014]. Yet, in the scientific understanding of spatial distribution of cities, it is mandatory to consider distributions other than hexagonal ones. This paper tries to exhaustively find bifurcating hexagonal and nonhexagonal patterns of spatial economy models on a hexagonal lattice. Moreover, as a novel finding of this paper, all these patterns are proved to be unstable just after bifurcation for whatever spatial economy models.

Considering the economic implication of bifurcating agglomeration patterns on the hexagonal lattice, we need to focus only on stable ones. This paper has been motivated by the following observations:

- In the infinite hexagonal lattice, all of the solution branches guaranteed by the equivariant branching lemma are, generically, unstable [Dionne et al., 1997].

\footnotetext{
*Author for correspondence

This is an Open Access article published by World Scientific Publishing Company. It is distributed under the terms of the Creative Commons Attribution 4.0 (CC-BY) License. Further distribution of this work is permitted, provided the original work is properly cited.
} 
- In the hexagonal lattice with periodic boundary conditions [Ikeda et al., 2017], there are stable nonhexagonal patterns, such as racetrack patterns. Bifurcating equilibria are always unstable just after bifurcation and often regain stability to become stable equilibria.

That said, this paper would like to make clear to what extent bifurcating equilibria on the lattice are unstable based on a bifurcation analysis of a scalar field of a finite groun $11 \mathrm{D}_{6} \dot{+}\left(\mathbb{Z}_{n} \times \mathbb{Z}_{n}\right)$. This paper would contribute to the study of a two-dimensional spatial agglomeration through the introduction of a methodology in group-theoretic bifurcation theory that has been developed mainly on the group $\mathrm{D}_{6}+\mathrm{T}^{2}\left(\mathrm{~T}^{2}\right.$ expresses the two-torus of translation symmetries). Whereas it is conventional in spatial economy to relate stability of bifurcating patterns to microeconomic modeling, this paper would search for model independent aspects of stability.

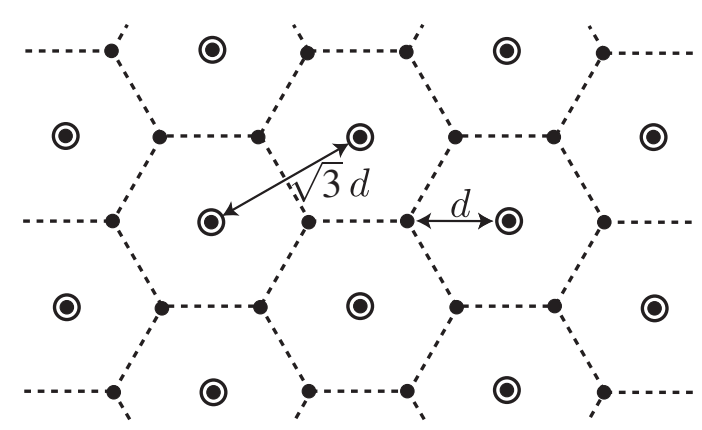

(a) $k=3$ system
Through this search, we would like to clarify the role of nonhexagonal patterns, in addition to hexagonal ones.

Central place theory in economic geography Christaller, 1933] was invented based on the distribution of cities and towns in southern Germany, which are spread out with geometrical orderliness. Figure 1 shows hexagonal market areas of three kinds proposed by Christaller. Lösch [1954] proposed a series of geometrically possible hexagons. Several attempts to simulate the self-organization of central place systems have been conducted through modeling of economic mechanisms. ${ }^{2}$ The geometry of cities was explained by fractals [Batty \& Longley, 1994. The distributions of satellite cities, towns, and villages around Berlin and London were shown to follow a universal law Makse et al., 1995]. Several studies of spatial agglomeration have been conducted on a square lattice Clarke \& Wilson, 1983; Weidlich \& Haag, 1987; Munz \& Weidlich, 1990;

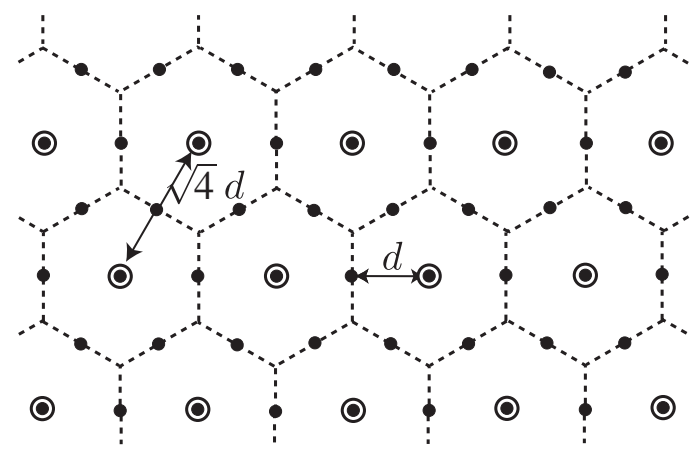

(b) $k=4$ system

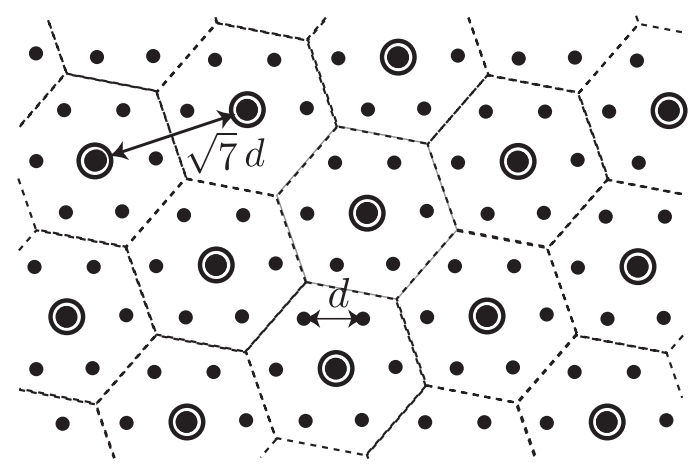

(c) $k=7$ system

Fig. 1. Three hexagonal systems predicted by Christaller 1933].

\footnotetext{
${ }^{1} \mathrm{D}_{6}$ is the dihedral group expressing local hexagonal symmetry and $\mathbb{Z}_{n}$ is a cyclic group.

${ }^{2}$ See, e.g. Eaton \& Lipsev, 1975; Clarke \& Wilson, 1983; Sanglier \& Allen, 1989; Munz \& Weidlich, 1990; Fujita et al., 1999a; Tabuchi \& Thisse, 2011; Banaszak et al., 2015].
} 
Brakman et al., 1999]. Stelder 2005 conducted a simulation of agglomeration for cities in Europe using a grid of points.

Hexagonal patterns have been observed for several physical phenomena, including the Bénard problem Bénard, 1900]. This problem was studied Sattinger, 1978] under a simplifying assumption that solutions are doubly periodic with respect to a hexagonal lattice. Mathematical analysis was conducted on the $\mathrm{D}_{6}+\mathrm{T}^{2}$-symmetric hexagonal lattice Buzano \& Golubitskv, 1983]. Equivariant bifurcation analysis of six- and 12-dimensional irreducible representations of this group has been conducted to search for possible bifurcated patterns: Hexagons, as well as rolls and triangles, exist for six-dimensional ones [Buzano \& Golubitsky, 1983; Dionne \& Golubitskv. 1992: Golubitsky \& Stewart, 2002]. Simple hexagons and super hexagons exist for 12-dimensional ones Kirchgässner. 1979: Dionne et al., 1997: Judd \& Silber, 2000].

Economic agglomeration patterns have been extensively studied in new economic geography. It is conventional to employ simple spatial platforms, such as two places and racetrack economy, in favor of their analytical tractability, whereas actual spatial agglomeration takes place in twodimensional spaces. Krugman [1996] regarded the racetrack economy, which comprises a system of identical cities spread uniformly around the circumference of a circle, as one-dimensional and inferred its extendibility to a two-dimensional economy to engender hexagonal distributions. In this economy, bifurcation produced a chain of spatially repeated core-periphery patterns a la Christaller and Lösch, which denotes a spatial alternation of a core place with a large population and a peripheral place with a small population 3

A hexagonal lattice has been used as a spatial platform of hexagonal distributions in central place theory. As an early attempt to provide central place theory with a microeconomic foundation, Eaton and Lipsey [1975] showed the existence of a hexagonal distribution of mobile production factors (e.g. firms and workers) by a partial equilibrium approach without referring to the stability of the hexagonal agglomeration. Hexagonal patterns of spatial economy models on a hexagonal lattice were shown to exist by theoretical and numerical studies of the bifurcation problem equivariant to $\mathrm{D}_{6} \dot{+}\left(\mathbb{Z}_{n} \times \mathbb{Z}_{n}\right)$ Ikeda et al. . 2012b; Ikeda et al., 2014; Ikeda \& Murota, 2014]. Not much attention, however, has been devoted to nonhexagonal patterns. It is a novel contribution of this paper to encompass nonhexagonal patterns, as well as hexagonal ones, and investigate their stability in a model independent manner.

This paper is organized as follows: Spatial economy models on a hexagonal lattice are introduced in Sec. 2. Bifurcation analysis procedure for the hexagonal lattice is presented in Sec. 3 Bifurcating patterns for $\mathrm{D}_{6} \dot{+}\left(\mathbb{Z}_{n} \times \mathbb{Z}_{n}\right)$-symmetric system are advanced and their stability is investigated in Sec. 4. Stability analysis for a spatial economy model is presented in Sec. 5

\section{A Spatial Economy Model on a Hexagonal Lattice}

We introduce in this section a spatial economy model on a hexagonal lattice comprising a system of uniformly distributed $n \times n$ places, and prescribe groups expressing the symmetry of this model.

\subsection{Hexagonal lattice}

We consider a rhombic, $n \times n$ hexagonal lattice with periodic boundary conditions: an example for $n=2$ is shown by the dashed lines in Fig. 2, A system of $n \times n$ places are allocated at hexagonal lattice points

$$
\mathbf{p}=n_{1} \ell_{1}+n_{2} \ell_{2}, \quad\left(n_{1}, n_{2}=0,1, \ldots, n-1\right)
$$

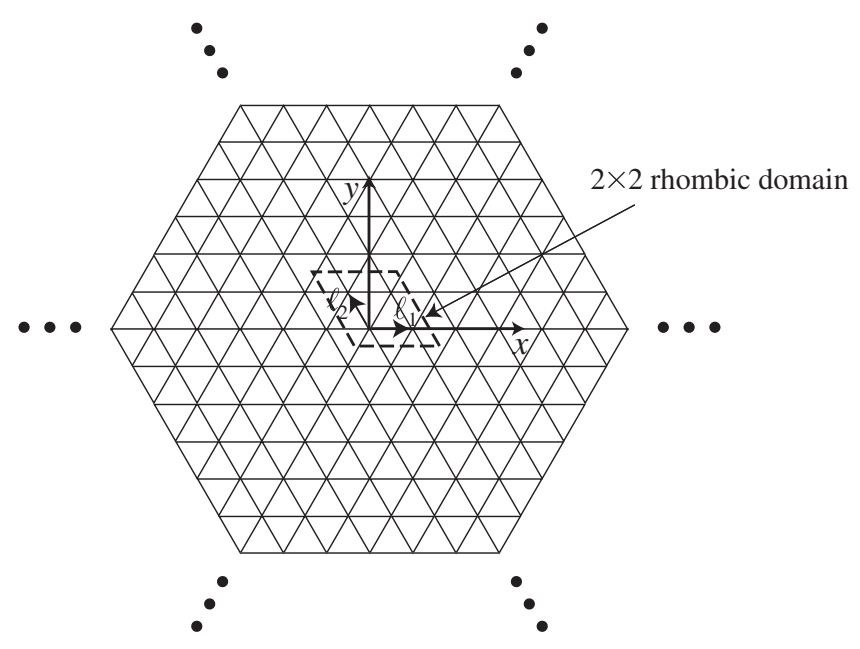

Fig. 2. Hexagonal lattice.

\footnotetext{
${ }^{3}$ See, e.g. [Krugman, 1996; ; Tabuchi \& Thisse, 2011; Ikeda et al., 2012a; Akamatsu et al., 2012].
} 
in a finite two-dimensional domain, where $\ell_{1}=$ $(d, 0)^{\top}$ and $\boldsymbol{\ell}_{2}=(-d / 2, d \sqrt{3} / 2)^{\top}$ are oblique basis vectors ( $d$ is the length of these vectors). Neighboring places, by the periodic boundary conditions, are connected by straight lines of equal length $d$ to form a regular-triangular mesh. These straight lines are interpreted as roads in a spatial economy model in Sec. 2.3 .

\subsection{Groups expressing the symmetry}

Symmetry of the $n \times n$ hexagonal lattice is described by the group

$$
G=\left\langle r, s, p_{1}, p_{2}\right\rangle=\mathrm{D}_{6} \dot{+}\left(\mathbb{Z}_{n} \times \mathbb{Z}_{n}\right),
$$

where $\langle\cdots\rangle$ denotes a group generated by the elements therein, $\dot{+}$ expresses the semidirect product, and

- $r$ : counterclockwise rotation about the origin at an angle of $\pi / 3$.

- $s$ : reflection $y \mapsto-y$.

- $p_{1}$ : periodic translation along the $\boldsymbol{\ell}_{1}$-axis (i.e. the $x$-axis).

- $p_{2}$ : periodic translation along the $\boldsymbol{\ell}_{2}$-axis.

The group $G$ contains the dihedral group $\langle r, s\rangle \simeq \mathrm{D}_{6}$ and cyclic groups $\left\langle p_{1}\right\rangle \simeq \mathbb{Z}_{n}$ and $\left\langle p_{2}\right\rangle \simeq \mathbb{Z}_{n}$ as its subgroups.

\subsection{Spatial economy model}

We employ a general spatial economy model [Fujita et al.,1999b]. The economy is composed of $K$ places (labeled $i=1, \ldots, K)$. Mobile populations are distributed over these places and are expressed by a vector $\boldsymbol{\lambda}=\left(\lambda_{1}, \ldots, \lambda_{K}\right)^{\top}\left(\lambda_{i} \geq 0\right)$. A transport cost is often chosen as a bifurcation parameter $\tau$. The utility $v_{i}$ is defined (often implicitly) as a function of $(\boldsymbol{\lambda}, \tau)$, that is, $\mathbf{v}=\mathbf{v}(\boldsymbol{\lambda}, \tau)$. This function is given by the microeconomic setting of the economy and varies with models (see, e.g. Sec. 5.2).

The governing equation of a spatial economy model is formulated in a standard form of static equilibrium as

$$
\mathbf{F}(\boldsymbol{\lambda}, \tau)=\mathbf{0}
$$

with

$$
F_{i}=\left(v_{i}-\bar{v}\right) \cdot \lambda_{i}
$$

and $\bar{v}=\sum_{j=1}^{K} v_{j} \lambda_{j}$. Therein $\mathbf{F}: \mathbb{R}^{K} \times \mathbb{R} \rightarrow \mathbb{R}^{K}$ is a sufficiently smooth nonlinear function in $\boldsymbol{\lambda}$ and $\tau$.

\section{Bifurcation Analysis Procedure for the Hexagonal Lattice}

Group-theoretic analysis procedure adapted to the $n \times n$ hexagonal lattice is presented in this section (see [1keda \& Murota, 2014] for theoretical details).

\subsection{Group-theoretic analysis procedure}

After a short introduction of basic concepts of group-theoretic bifurcation theory, group-theoretic analysis procedure is presented. The symmetry of a system of $n \times n$ places on the hexagonal lattice is described by the equivariance of $\mathbf{F}(\boldsymbol{\lambda}, \tau)$ to $G$ as

$$
T(g) \mathbf{F}(\boldsymbol{\lambda}, \tau)=\mathbf{F}(T(g) \boldsymbol{\lambda}, \tau), \quad g \in G,
$$

in terms of an orthogonal matrix representation $T$ of group $G=\left\langle r, s, p_{1}, p_{2}\right\rangle$ in (1) on the $K$ dimensional space $\mathbb{R}^{K}$ for $K=n^{2}$. Each element $g$ of $G$ in (1) acts as a permutation among place numbers $(1, \ldots, K)$ and hence each $T(g)$ is a permutation matrix. Then we can show the equivariance (4) of a spatial economy model to $G$ [Ikeda \& Murota, 2014, p. 114].

Consider a critical point $\left(\boldsymbol{\lambda}_{c}, \tau_{c}\right)$ of multiplicity $M(\geq 1)$, at which the Jacobian matrix $J=\partial \mathbf{F} / \partial \boldsymbol{\lambda}$ of $\mathbf{F}$ has $M$ zero eigenvalues 5 Each critical point can be associated with an irreducible representation $\mu$ of the group $G$. Using a standard procedure called the Lyapunov-Schmidt reduction with symmetry Golubitsky et al., 1988; Ikeda \& Murota, 2010], the full system of equations $\mathbf{F}(\boldsymbol{\lambda}, \tau)=\mathbf{0}$ in $\lambda \in \mathbb{R}^{K}$ [see (2)] is reduced, in a neighborhood of $\left(\boldsymbol{\lambda}_{c}, \tau_{c}\right)$, to a system of $M$ equations (called bifurcation equations)

$$
\tilde{\mathbf{F}}(\mathbf{w}, \tilde{\tau})=\mathbf{0}
$$

in $\mathbf{w} \in \mathbb{R}^{M}$, where $\tilde{\mathbf{F}}: \mathbb{R}^{M} \times \mathbb{R} \rightarrow \mathbb{R}^{M}$ is a function and $\tilde{\tau}=\tau-\tau_{c}$ denotes the increment of $\tau$. The equivariance of the full system, which is formulated

\footnotetext{
${ }^{4}$ The form in (3) is associated with the replicator dynamics that is customarily used in spatial economics (e.g. [Fujita et al., $1999 \mathrm{~b})$.

${ }^{5}$ Throughout this paper we assume that a critical point is generic (or group-theoretic) in the sense that the $M$-dimensional kernel space of the Jacobian matrix is irreducible with respect to the representation $T$.
} 
in (44), is inherited by the reduced system (5) in the following form:

$$
\tilde{T}(g) \tilde{\mathbf{F}}(\mathbf{w}, \tilde{\tau})=\tilde{\mathbf{F}}(\tilde{T}(g) \mathbf{w}, \tilde{\tau}), \quad g \in G,
$$

where $\tilde{T}$ is the subrepresentation of $T$ on the $M$ dimensional kernel space of the Jacobian matrix. The reduced equation (5) is to be solved for $\mathbf{w}$ as $\mathbf{w}=\mathbf{w}(\tilde{\tau})$ with respect to the symmetry condition of $\tilde{\mathbf{F}}$ described in (6). Each $\mathbf{w}$ uniquely determines a solution $\boldsymbol{\lambda}$ of the full system (2).

The group-theoretic bifurcation analysis to investigate the stability of bifurcating solutions of a critical point proceeds as follows:

Step 1. Specify an irreducible representation.

Step 2. Obtain the expanded form of the bifurcation equation by exploiting the symmetry.

Step 3. Obtain bifurcating solutions by solving this equation.

Step 4. Obtain the Jacobian matrix of the bifurcation equation on the bifurcating solutions to determine their stability.

After the introduction of irreducible representations of the group $G$ in Sec. 3.2, these steps are explained using a simple example in Sec. 3.3 .

\subsection{Irreducible representations}

The uniform distribution on the $n \times n$ hexagonal lattice is given by $\boldsymbol{\lambda}=\frac{1}{n^{2}}(1, \ldots, 1)^{\top}$, and its symmetry is labeled by the group $G=\left\langle r, s, p_{1}, p_{2}\right\rangle=$ $\mathrm{D}_{6} \dot{+}\left(\mathbb{Z}_{n} \times \mathbb{Z}_{n}\right)$ in (1). In Step 1 of the analysis, an irreducible representation of $G$ is specified. By virtue of the consideration of a spatial economy model that has a single scalar variable at each node of the lattice, there are limited irreducible representations, called $\mu$, and, in turn, limited bifurcating patterns from a uniform state in comparison with those given in the literature Golubitsky \& Stewart, 2002 .

The whole set $R(G)$ of these irreducible representations $\mu$ was obtained Ikeda \& Murota, 2014, p. 187] a:6

$$
R(G)=\{(1),(2),(3),(6 ; k, 0),(6 ; k, k),(12 ; k, \ell)\} .
$$

For $\mu$ being expressed as $(M)$ or $(M ; k, \ell), M$ stands for the multiplicity of the critical point and $k$ and $\ell$ mean wave numbers of the critical eigenvectors. The multiplicity $M$ of the critical point, accordingly, is generically either $1,2,3,6$, or 12 . The irreducible representation $\mu=(1)$, for example, is defined by

$$
\begin{aligned}
& T^{(1)}(r)=1, \quad T^{(1)}(s)=1, \\
& T^{(1)}\left(p_{1}\right)=1, \quad T^{(1)}\left(p_{2}\right)=1
\end{aligned}
$$

and other irreducible representations are defined in Appendix A.

\subsection{Simple example of bifurcation and stability analysis}

The bifurcation and stability analysis represented in Sec. 3.1 is demonstrated step by step for a simple example.

Step 1. The targeted irreducible representation is specified, for example, as $\mu=(3)$.

Step 2. The equivariance of the bifurcation equation to the group $\mathrm{D}_{6} \ltimes\left(\mathbb{Z}_{n} \times \mathbb{Z}_{n}\right)$ is identical to the equivariance to the action of the four elements $r, s, p_{1}, p_{2}$ generating this group. Therefore, the equivariance condition (6) of the bifurcation equation (5) can be expressed as Ikeda \& Murota, 2014, Sec. 9.4.2]

$$
\begin{aligned}
& r: \tilde{F}_{3}\left(w_{1}, w_{2}, w_{3}\right)=\tilde{F}_{1}\left(w_{3}, w_{1}, w_{2}\right), \\
& \tilde{F}_{1}\left(w_{1}, w_{2}, w_{3}\right)=\tilde{F}_{2}\left(w_{3}, w_{1}, w_{2}\right), \\
& \tilde{F}_{2}\left(w_{1}, w_{2}, w_{3}\right)=\tilde{F}_{3}\left(w_{3}, w_{1}, w_{2}\right), \\
& s: \tilde{F}_{3}\left(w_{1}, w_{2}, w_{3}\right)=\tilde{F}_{1}\left(w_{3}, w_{2}, w_{1}\right), \\
& \tilde{F}_{2}\left(w_{1}, w_{2}, w_{3}\right)=\tilde{F}_{2}\left(w_{3}, w_{2}, w_{1}\right), \\
& \tilde{F}_{1}\left(w_{1}, w_{2}, w_{3}\right)=\tilde{F}_{3}\left(w_{3}, w_{2}, w_{1}\right), \\
& p_{1}:-\tilde{F}_{1}\left(w_{1}, w_{2}, w_{3}\right)=\tilde{F}_{1}\left(-w_{1}, w_{2},-w_{3}\right), \\
& \tilde{F}_{2}\left(w_{1}, w_{2}, w_{3}\right)=\tilde{F}_{2}\left(-w_{1}, w_{2},-w_{3}\right), \\
&-\tilde{F}_{3}\left(w_{1}, w_{2}, w_{3}\right)=\tilde{F}_{3}\left(-w_{1}, w_{2},-w_{3}\right),
\end{aligned}
$$

$\overline{{ }^{6} \text { The irreducible representations that were called }}(1 ;+,+),(2 ;+),(3 ;+,+),(6 ; k, 0 ;+)$, and $(6 ; k, k ;+)$ in Ikeda \& Murota, 2014 are simplified herein to (1), (2), (3), $(6 ; k, 0)$, and $(6 ; k, k)$, respectively. $\mu=(2)$ exists when $n$ is a multiple of 3 and $\mu=(3)$ exists when $n$ is even. $k$ for $(6 ; k, 0)$ runs over $1 \leq k \leq\left\lfloor\frac{n-1}{2}\right\rfloor$, where $\lfloor x\rfloor$ denotes the largest integer not larger than $x$, for a real number $x . k$ for $(6 ; k, k)$ runs over $1 \leq k \leq\left\lfloor\frac{n-1}{2}\right\rfloor, k \neq \frac{n}{3}$ and $(k, \ell)$ for $(12 ; k, \ell)$ runs over $1 \leq \ell \leq k-1$ and $2 k+\ell \leq n-1$. 


$$
\begin{aligned}
& p_{2}: \tilde{F}_{1}\left(w_{1}, w_{2}, w_{3}\right)=\tilde{F}_{1}\left(w_{1},-w_{2},-w_{3}\right), \\
&-\tilde{F}_{2}\left(w_{1}, w_{2}, w_{3}\right)=\tilde{F}_{2}\left(w_{1},-w_{2},-w_{3}\right), \\
&-\tilde{F}_{3}\left(w_{1}, w_{2}, w_{3}\right)=\tilde{F}_{3}\left(w_{1},-w_{2},-w_{3}\right) .
\end{aligned}
$$

Then a set of bifurcation equations $\left(\tilde{F}_{i} \mid i=1,2,3\right)$ takes the form:

$$
\begin{aligned}
& \tilde{F}_{1}\left(w_{1}, w_{2}, w_{3}, \tilde{\tau}\right)=\tilde{F}_{2}\left(w_{3}, w_{1}, w_{2}, \tilde{\tau}\right), \\
& \tilde{F}_{2}\left(w_{1}, w_{2}, w_{3}, \tilde{\tau}\right) \\
& \quad=w_{2} \sum_{a=0} \sum_{b=0} \sum_{c=0} A_{2 a, 2 b+1,2 c}(\tilde{\tau}) w_{1}^{2 a} w_{2}^{2 b} w_{3}^{2 c} \\
& \quad+w_{1} w_{3} \sum_{a=0} \sum_{b=0} \sum_{c=0} A_{2 a+1,2 b, 2 c+1}(\tilde{\tau}) w_{1}^{2 a} w_{2}^{2 b} w_{3}^{2 c},
\end{aligned}
$$

$\tilde{F}_{3}\left(w_{1}, w_{2}, w_{3}, \tilde{\tau}\right)=\tilde{F}_{2}\left(w_{2}, w_{3}, w_{1}, \tilde{\tau}\right)$

with all coefficients $A_{2 a, 2 b+1,2 c}$ and $A_{2 a+1,2 b, 2 c+1}$ being real.

Step 3. We choose the basis vectors $\mathbf{q}_{i}^{(3)}(i=1,2,3)$ of the three-dimensional space of $\mathbf{w}$ so as to represent stripes in three different directions, as depicted in Fig. 3(a) (see Sec. 3.4 for details). The components $w_{1}, w_{2}$, and $w_{3}$ of $\mathbf{w}$ are chosen so as to be related to $\mathbf{q}_{1}^{(3)}, \mathbf{q}_{2}^{(3)}$, and $\mathbf{q}_{3}^{(3)}$, respectively. Then we note that a system of bifurcation equations $\tilde{F}_{1}=\tilde{F}_{2}=\tilde{F}_{3}=0$ has the following bifurcating solutions:

- the stripe pattern $\mathbf{w}_{\text {stripe }}=(w, 0,0)[\mathrm{Fig}$. 3(a)],

- the hexagonal pattern $\mathbf{w}_{\text {hexa }}=(w, w, w)$

[Fig. 3(b)], and
- the racetrack pattern $\mathbf{w}_{\text {racetrack }}=(-w,-w,-w)$ [Fig. 3(c)].

Step 4. For the hexagonal pattern $\mathbf{w}_{\text {hexa }}=$ $(w, w, w)$, we have

$$
\begin{aligned}
\tilde{F}_{1}(w, w, w, \tilde{\tau}) & =\tilde{F}_{2}(w, w, w, \tilde{\tau}) \\
& =\tilde{F}_{3}(w, w, w, \tilde{\tau}) \\
& \approx A_{010}^{\prime}(0) \tilde{\tau}+A_{101}(0) w,
\end{aligned}
$$

where $A_{010}^{\prime}(0)=\partial A_{010} / \partial \tilde{\tau}(0)$ is generically nonzero.7 Hence the bifurcating solutions takes an asymptotic form: $\tilde{\tau}=\tilde{\tau}_{1}(w) \approx-\frac{A_{101}(0) w}{A_{010}^{\prime}(0)}$ and the Jacobian matrix [cf. $(\underline{B .2})]$ becomes

$$
\tilde{J}\left(\mathbf{w}_{\text {hexa }}, \tilde{\tau}_{1}\right) \approx A_{101}(0) w\left(\begin{array}{rrr}
-1 & 1 & 1 \\
1 & -1 & 1 \\
1 & 1 & -1
\end{array}\right) .
$$

The eigenvalues of this Jacobian matrix are evaluated to $\lambda_{1} \approx A_{101}(0) w$ and $\lambda_{2} \approx-2 A_{101}(0) w$ (repeated twice). Thus $\lambda_{1}$ and $\lambda_{2}$ have opposite signs; accordingly, the hexagonal pattern is always unstable. The racetrack pattern can be shown as unstable by replacing $w$ with $-w$ in the discussion above. The stripe pattern is also unstable as shown in Appendix B.2.

\subsection{Eigenvectors of the Jacobian matrix}

By virtue of the symmetry (4), the eigenvectors of the Jacobian matrix $J=\partial \mathbf{F} / \partial \boldsymbol{\lambda}$ can be obtained analytically as explained below. We consider a transformation matrix $Q$ that is made up of submatrices $Q^{\mu}(\mu \in R(G))$, i.e. $Q=\left[\ldots, Q^{\mu}, \ldots\right]$. Each

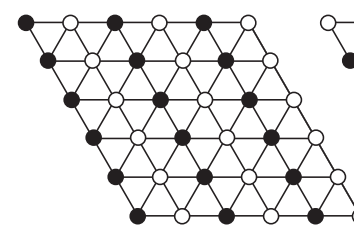

$\boldsymbol{q}_{1}^{(3)}$

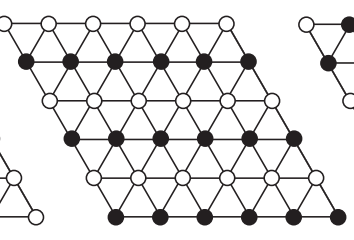

$\boldsymbol{q}_{2}^{(3)}$

(a)

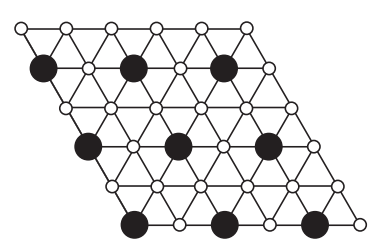

$$
\boldsymbol{q}_{1}^{(3)}+\boldsymbol{q}_{2}^{(3)}+\boldsymbol{q}_{3}^{(3)}
$$

(b)

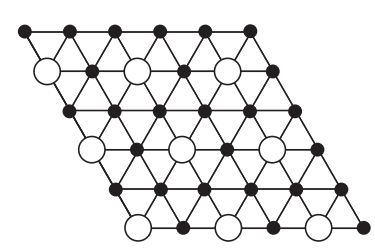

$$
-\boldsymbol{q}_{1}^{(3)}-\boldsymbol{q}_{2}^{(3)}-\boldsymbol{q}_{3}^{(3)}
$$

(c)

Fig. 3. Spatial patterns expressed by the basis vectors $\mathbf{q}_{i}^{(3)}(i=1,2,3)$ on the $6 \times 6$ hexagonal lattice (the black circles indicate positive components; the white circles indicate negative components). (a) Stripe patterns, (b) hexagonal pattern and (c) racetrack pattern.

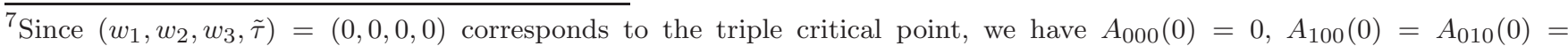
$A_{001}(0)=0$ and $A_{010}(\tilde{\tau}) \approx A_{010}^{\prime}(0) \tilde{\tau}$.
} 
submatrix consists of the eigenvectors $\mathbf{q}_{i}^{\mu}$ of $J$, i.e.

$$
Q^{\mu}=\left(\mathbf{q}_{i}^{\mu} \mid i=1, \ldots, M(\mu)\right) .
$$

As presented in Remark 3.1 below, the concrete forms of $\mathbf{q}_{i}^{\mu}$ in $Q^{\mu}$ are given by discrete cosine and sine series, and are independent on the economic modeling of the equilibrium condition (2).

The transformation matrix $Q$ puts the Jacobian matrix $J$ into a diagonal form:

$$
Q^{\top} J Q=\operatorname{diag}\left(\ldots, \eta^{\mu} I_{M(\mu)}, \ldots\right), \quad \mu \in R(G),
$$

where $I_{M(\mu)}$ is the $M(\mu) \times M(\mu)$ identity matrix and $\operatorname{diag}(\cdots)$ denotes a block diagonal matrix with diagonal entries therein. Bifurcating patterns from the uniform state are self-organized when some eigenvalue $\eta^{\mu}=\left(\mathbf{q}_{i}^{\mu}\right)^{\top} J \mathbf{q}_{i}^{\mu}$ (repeated $M(\mu)$ times) becomes zero and each $\mu$ labels different bifurcating pattern(s).

Remark 3.1. The submatrices $Q^{\mu}$ of the transformation matrix $Q$ for the economy on the $n \times n$ hexagonal lattice are given by Ikeda \& Murota, 2014, Chapter 7]:

$$
\begin{aligned}
& Q^{(1)}=\mathbf{q}_{1}^{(1)}=\frac{1}{n}(1, \ldots, 1)^{\top}=\langle 1\rangle, \\
& Q^{(2)}=\left[\mathbf{q}_{1}^{(2)}, \mathbf{q}_{2}^{(2 ;+)}\right]=\left[\left\langle\cos \left(\frac{2 \pi\left(n_{1}-2 n_{2}\right)}{3}\right)\right\rangle,\left\langle\sin \left(\frac{2 \pi\left(n_{1}-2 n_{2}\right)}{3}\right)\right\rangle\right], \\
& Q^{(3)}=\left[\mathbf{q}_{1}^{(3)}, \mathbf{q}_{2}^{(3)}, \mathbf{q}_{3}^{(3)}\right]=\left[\left\langle\cos \left(\pi n_{1}\right)\right\rangle,\left\langle\cos \left(\pi n_{2}\right)\right\rangle,\left\langle\cos \left(\pi\left(n_{1}-n_{2}\right)\right)\right\rangle\right], \\
& Q^{(6 ; k, 0)}=\left[\mathbf{q}_{1}^{(6 ; k, 0)}, \ldots, \mathbf{q}_{6}^{(6 ; k, 0)}\right] \\
& =\left[\left\langle\cos \left(\frac{2 \pi k n_{1}}{n}\right)\right\rangle,\left\langle\sin \left(\frac{2 \pi k n_{1}}{n}\right)\right\rangle,\left\langle\cos \left(\frac{2 \pi k\left(-n_{2}\right)}{n}\right)\right\rangle,\left\langle\sin \left(\frac{2 \pi k\left(-n_{2}\right)}{n}\right)\right\rangle,\right. \\
& \left.\left\langle\cos \left(\frac{2 \pi k\left(-n_{1}+n_{2}\right)}{n}\right)\right\rangle,\left\langle\sin \left(\frac{2 \pi k\left(-n_{1}+n_{2}\right)}{n}\right)\right\rangle\right] \\
& Q^{(6 ; k, k)}=\left[\mathbf{q}_{1}^{(6 ; k, k)}, \ldots, \mathbf{q}_{6}^{(6 ; k, k)}\right] \\
& =\left[\left\langle\cos \left(\frac{2 \pi k\left(n_{1}+n_{2}\right)}{n}\right)\right\rangle,\left\langle\sin \left(\frac{2 \pi k\left(n_{1}+n_{2}\right)}{n}\right)\right\rangle,\left\langle\cos \left(\frac{2 \pi k\left(n_{1}-2 n_{2}\right)}{n}\right)\right\rangle,\right. \\
& \left.\left\langle\sin \left(\frac{2 \pi k\left(n_{1}-2 n_{2}\right)}{n}\right)\right\rangle,\left\langle\cos \left(\frac{2 \pi k\left(-2 n_{1}+n_{2}\right)}{n}\right)\right\rangle,\left\langle\sin \left(\frac{2 \pi k\left(-2 n_{1}+n_{2}\right)}{n}\right)\right\rangle\right], \\
& Q^{(12 ; k, \ell)}=\left[\mathbf{q}_{1}^{(12 ; k, \ell)}, \ldots, \mathbf{q}_{12}^{(12 ; k, \ell)}\right] \\
& =\left[\left\langle\cos \left(\frac{2 \pi\left(k n_{1}+\ell n_{2}\right)}{n}\right)\right\rangle,\left\langle\sin \left(\frac{2 \pi\left(k n_{1}+\ell n_{2}\right)}{n}\right)\right\rangle,\left\langle\cos \left(\frac{2 \pi\left(\ell n_{1}-(k+\ell) n_{2}\right)}{n}\right)\right\rangle,\right. \\
& \left\langle\sin \left(\frac{2 \pi\left(\ell n_{1}-(k+\ell) n_{2}\right)}{n}\right)\right\rangle,\left\langle\cos \left(\frac{2 \pi\left(-(k+\ell) n_{1}+k n_{2}\right)}{n}\right)\right\rangle, \\
& \left\langle\sin \left(\frac{2 \pi\left(-(k+\ell) n_{1}+k n_{2}\right)}{n}\right)\right\rangle,\left\langle\cos \left(\frac{2 \pi\left(k n_{1}-(k+\ell) n_{2}\right)}{n}\right)\right\rangle, \\
& \left\langle\sin \left(\frac{2 \pi\left(k n_{1}-(k+\ell) n_{2}\right)}{n}\right)\right\rangle,\left\langle\cos \left(\frac{2 \pi\left(\ell n_{1}+k n_{2}\right)}{n}\right)\right\rangle,\left\langle\sin \left(\frac{2 \pi\left(\ell n_{1}+k n_{2}\right)}{n}\right)\right\rangle, \\
& \left.\left\langle\cos \left(\frac{2 \pi\left(-(k+\ell) n_{1}+\ell n_{2}\right)}{n}\right)\right\rangle,\left\langle\sin \left(\frac{2 \pi\left(-(k+\ell) n_{1}+\ell n_{2}\right)}{n}\right)\right\rangle\right] .
\end{aligned}
$$


Here, for a vector $\left(g\left(n_{1}, n_{2}\right) \mid n_{1}, n_{2}=0,1, \ldots, n-1\right)$ on the lattice, we use the notation $\left\langle g\left(n_{1}, n_{2}\right)\right\rangle$ for its normalization $\left(\left\|\left\langle g\left(n_{1}, n_{2}\right)\right\rangle\right\|=1\right)$.

\section{Theoretically Predicted Bifurcating Distributions and Their Stability}

Self-organizing bifurcating patterns from a uniform state associated with $\lambda=\frac{1}{K}(1, \ldots, 1)^{\top}$ are investigated by group-theoretic bifurcation theory. Whereas Ikeda and Murota 2014] obtained all hexagonal patterns and some stripe patterns of the hexagonal lattice, this paper advances more patterns, such as racetrack patterns, other stripe patterns, and upside-down patterns. Although the procedure to find these patterns is heuristic, the authors endeavored to find all bifurcating patterns.

\subsection{Bifurcating patterns}

By the stepwise procedure presented in Sec. 3.1 we investigated bifurcating equilibria and their stability for each $\mu$, as worked out in Appendix B. We relate the components of $\mathbf{w}$ to the column vectors of $Q^{\mu}$.

The hexagonal bifurcating patterns are associated with 8

$$
\mathbf{w}=\mathbf{w}_{\text {hexa }}= \begin{cases}(w, 0) & \text { for } \mu=(2), \\ (w, w, w) & \text { for } \mu=(3), \\ (w, 0, w, 0, w, 0) & \text { for } \mu=(6 ; k, \ell)(\ell=0, k), \\ (w, 0, w, 0, w, 0, w, 0, w, 0, w, 0) & \text { for } \mu=(12 ; k, \ell)(\text { Type VM), } \\ (w, 0, w, 0, w, 0,0,0,0,0,0,0) & \text { for } \mu=(12 ; k, \ell) \text { (Type T). }\end{cases}
$$

Hexagonal patterns on the $6 \times 6$ hexagonal lattice are depicted in Fig. 廿(a). The existence of the bifurcating hexagonal solution of Type $\mathrm{T}$ (tilted hexagon) is conditional on the values of $k, \ell$, and $n$ as expounded in Proposition 1 Ikeda \& Murota, 2014]. For example, Type $\mathrm{T}$ hexagon exists for $\mu=(12 ; 2,1)$ on the $7 \times 7$ hexagonal lattice (Fig. 5 ). The racetrack bifurcating patterns are associated with the reversed directions of hexagonal bifurcating patterns. That is, $\mathbf{w}_{\text {racetrack }}=-\mathbf{w}_{\text {hexa }}$.

Proposition 1. A hexagonal pattern $\mathbf{w}_{\text {hexaT }}$ of Type T exists when

$$
\begin{array}{r}
(\hat{k}-\hat{\ell}) \operatorname{gcd}(\hat{k}, \hat{\ell}) \text { is not divisible by } \\
\operatorname{gcd}\left(\hat{k}^{2}+\hat{k} \hat{\ell}+\hat{\ell}^{2}, \hat{n}\right) .
\end{array}
$$

Here

$$
\begin{aligned}
& \hat{k}=\frac{k}{\operatorname{gcd}(k, \ell, n)}, \\
& \hat{\ell}=\frac{\ell}{\operatorname{gcd}(k, \ell, n)}, \\
& \hat{n}=\frac{n}{\operatorname{gcd}(k, \ell, n)},
\end{aligned}
$$

and $\operatorname{gcd}(k, \ell, n)$ means the greatest common divisor of $k, \ell$, and $n$.

Proof. See Proposition 8.28 in Ikeda \& Murota, 2014, p. 252].

There are stripe-like patterns that are associated with

for $\mu=(3)$,

for $\mu=(6 ; k, 0),(6 ; k, k)$ (Type I),

for $\mu=(6 ; k, 0),(6 ; k, k)$ (Type II),

for $\mu=(12 ; k, \ell)$ (Type I),

for $\mu=(12 ; k, \ell)$ (Type II).

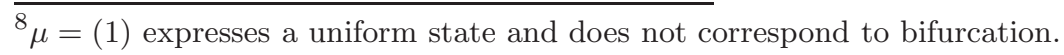



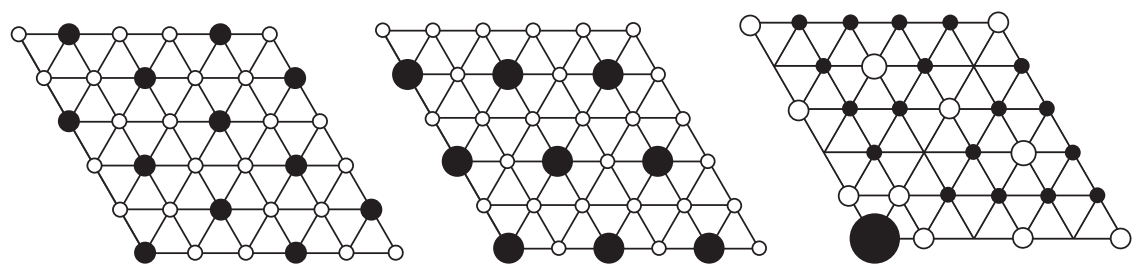

$\boldsymbol{q}_{1}^{(2)}$

$\boldsymbol{q}_{1}^{(3)}+\boldsymbol{q}_{2}^{(3)}+\boldsymbol{q}_{3}^{(3)}$

$\sum_{i=1}^{6} \boldsymbol{q}_{2 i-1}^{(12 ; 2,1)}$
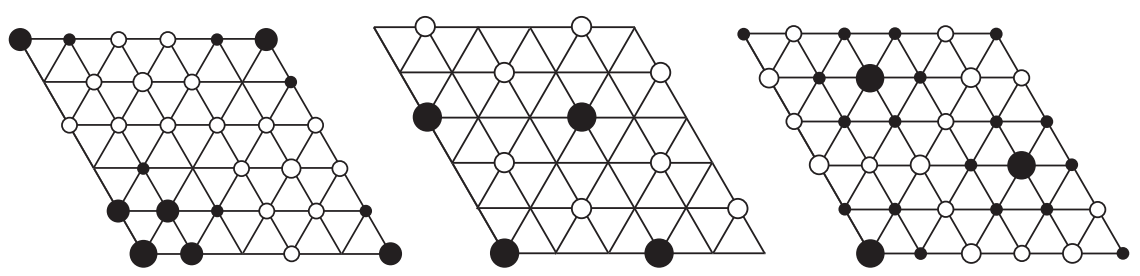

$\sum_{i=1}^{3} \boldsymbol{q}_{2 i-1}^{(6 ; 1,0)}$

$\sum_{i=1}^{3} \boldsymbol{q}_{2 i-1}^{(6 ; 2,0)}$

$\sum_{i=1}^{3} \boldsymbol{q}_{2 i-1}^{(6 ; 1,1)}$

(a)
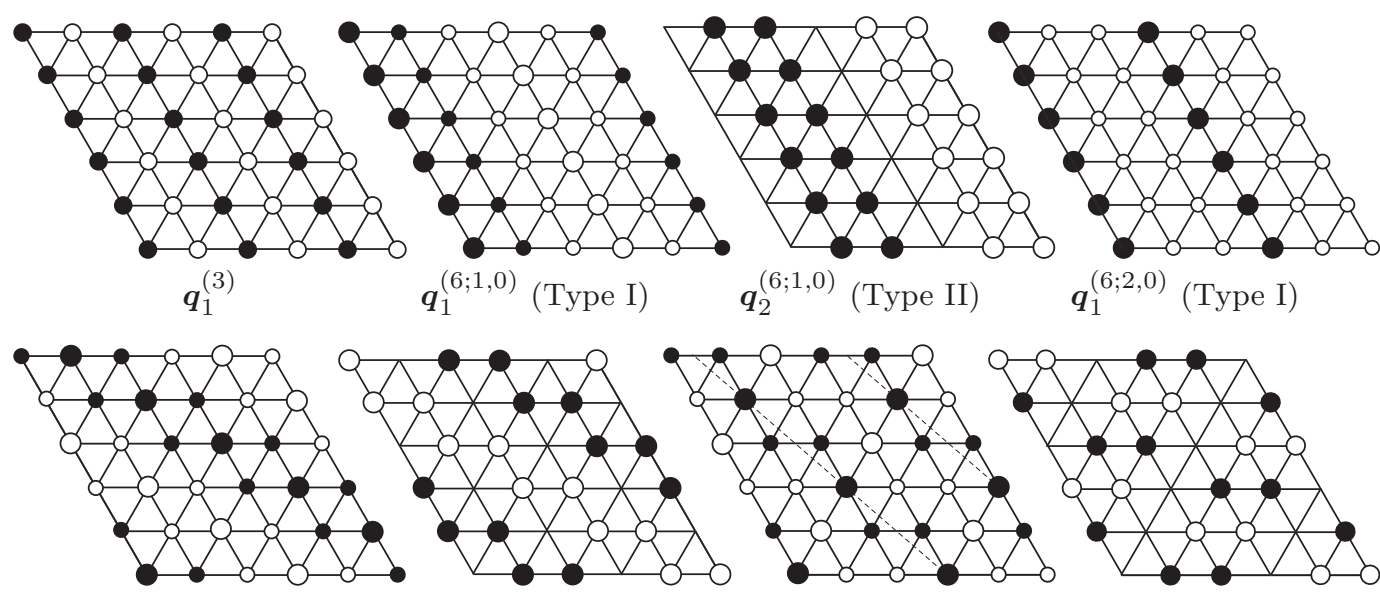

$\boldsymbol{q}_{1}^{(6 ; 1,1)}$ (Type I)

$\boldsymbol{q}_{2}^{(6 ; 1,1)}$ (Type II)
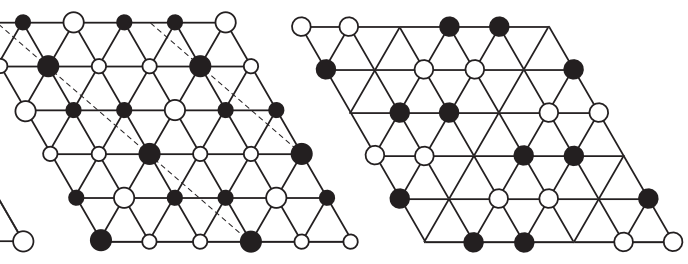

$\boldsymbol{q}_{1}^{(12 ; 2,1)}($ Type I)

$\boldsymbol{q}_{2}^{(12 ; 2,1)}$ (Type II)

(b)

Fig. 4. Eigenvectors for bifurcating hexagonal and stripe patterns on the $6 \times 6$ hexagonal lattice (the black circles indicate positive components; the white circles indicate negative components). (a) Hexagonal patterns and (b) stripe patterns.

Stripe patterns on the $6 \times 6$ hexagonal lattice are depicted in Fig. 4 (b). The existence of some bifurcating stripe solutions is conditional on the values of $k, \ell$, and $n$ as expounded in Propositions 2]and 3 .

Proposition 2. Type II stripe solution exists when $\hat{n}$ is even for $\mu=(6 ; k, 0)$ and when $\tilde{n}$ is even for $\mu=(6 ; k, k)$. Here

$\hat{n}=\frac{n}{\operatorname{gcd}(k, n)}, \quad \tilde{n}= \begin{cases}\frac{\hat{n}}{3} & \text { if } \hat{n} \text { is a multiple of } 3, \\ \hat{n} & \text { otherwise. }\end{cases}$

Proof. See Sec. B.3.3 in Appendix B.3.

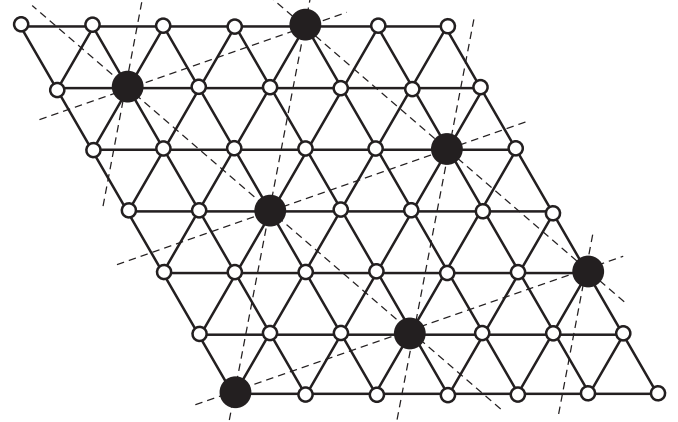

Fig. 5. Type $\mathrm{T}$ hexagon for $\mu=(12 ; 2,1)$ on the $7 \times 7$ hexagonal lattice (the black circles indicate positive components; the white circles indicate negative components). 
Proposition 3. For $\mu=(12 ; k, \ell)$, Type I stripe solution exists when the condition

$$
\begin{array}{r}
\hat{k}^{2}+\hat{k} \hat{\ell}+\hat{\ell}^{2}, \hat{k}^{2}+2 \hat{k} \hat{\ell}, \hat{k}^{2}-\hat{\ell}^{2} \text {, and } \\
\hat{l}^{2}+2 \hat{k} \hat{\ell} \text { are not divisible by } \hat{n}
\end{array}
$$

is satisfied. Type II stripe solution exists when the condition (12) is satisfied and $\hat{n}$ is even.

Proof. See Appendix C.1.

There are upside-down symmetric patterns that possibly appear for $\mu=(12 ; k, \ell)$ associated with

$$
\begin{aligned}
& \mathbf{w}_{\text {upside-down }} \\
& \quad= \begin{cases}(w, 0,0,0,0,0, w, 0,0,0,0,0) & (\text { Type I }), \\
(0, w, 0,0,0,0,0, w, 0,0,0,0) & \text { (Type II). }\end{cases}
\end{aligned}
$$

The existence of this pattern $\mathbf{w}_{\text {upside-down for } \mu=}$ $(12 ; k, \ell)$ is conditional on the values of $k, \ell$, and $n$, as expounded in Proposition 4 (see Appendix C.2 for the proof). Its existence is relatively rare in comparison with that of other patterns 9

Proposition 4. Type I upside-down pattern for $\mu=$ $(12 ; k, \ell)$ exists when the condition

$$
\begin{aligned}
& (\hat{k}-\hat{\ell}) \operatorname{gcd}(\hat{k}, \hat{\ell}) \text { is not divisible by } \\
& \quad \operatorname{gcd}\left(\hat{k}^{2}+2 \hat{k} \hat{\ell}, \hat{n}\right) \text { and } \operatorname{gcd}\left(\hat{k}^{2}+\hat{k} \hat{\ell}+\hat{\ell}^{2}, \hat{n}\right)
\end{aligned}
$$

is satisfied. Type II upside-down pattern exists when $\hat{n}$ is even and this condition is satisfied.

\subsection{Stability of bifurcating solutions}

We would like to advance a bold statement on the stability of bifurcating solutions from the uniform state of the hexagonal lattice. An amazing structure of the eigenvalues of the Jacobian matrix for bifurcating solutions is that there are opposite signs of eigenvalues (e.g. Appendices B.1-B.3). This structure guarantees that the bifurcating solutions are unstable. The following proposition gives a rich implication on the stability of bifurcating solutions (see Sec. 3.3 and Appendix B for the proof).
Proposition 5. The hexagonal, racetrack, stripe and upside-down patterns are asymptotically unstable in the neighborhood of the bifurcation point of a uniform state.

This proposition is in line with the results of infinite hexagonal lattice that "all of the solution branches guaranteed by the equivariant branching lemma are, generically, unstable" Dionne et al., 1997.

\section{Numerical Bifurcation Analysis of a Spatial Economy Model}

A numerical bifurcation analysis is conducted on a spatial economy model on a $6 \times 6$ hexagonal lattice. Bifurcating solution curves are obtained and their stability is investigated with reference to theoretical results in Sec. 4

\subsection{Bifurcation mechanism}

This lattice is invariant to the group $G=$ $\left\langle r, s, p_{1}, p_{2}\right\rangle=\mathrm{D}_{6} \dot{+}\left(\mathbb{Z}_{6} \times \mathbb{Z}_{6}\right)$ in (11). The set of irreducible representations for this $6 \times 6$ hexagonal lattice is given by (7) as

$$
\begin{aligned}
& R(G)=\{(1),(2),(3),(6 ; 1,0),(6 ; 2,0), \\
&(6 ; 1,1),(12 ; 2,1)\} .
\end{aligned}
$$

The bifurcating patterns are listed in Table 1

There are hexagonal patterns of Type VM, racetrack patterns and stripe patterns, whereas a Type $\mathrm{T}$ hexagon and an upside-down pattern are absent (Remark 5.1).

Remark 5.1. First, for $(6,2,0)$, we have $\hat{n}=3$ and $\tilde{n}=1$; since $\tilde{n}$ is odd, Type II stripe does not exist (Proposition 2). For $(12 ; 2,1)$ with $n=6$, we have $(\hat{k}, \hat{\ell}, \hat{n})=(2,1,6)$ and Type $\mathrm{T}$ hexagon does not exist because $(\hat{k}-\hat{\ell}) \operatorname{gcd}(\hat{k}, \hat{\ell})=1$ is divisible by $\operatorname{gcd}\left(\hat{k}^{2}+\hat{k} \hat{\ell}+\hat{\ell}^{2}, \hat{n}\right)=\operatorname{gcd}(7,6)=1$ (Proposition 11). Both stripe patterns exist because $\hat{n}$ is even and $\hat{k}^{2}+\hat{k} \hat{\ell}+\hat{\ell}^{2}=7, \hat{k}^{2}+2 \hat{k} \hat{\ell}=8$, $k^{2}-\hat{\ell}^{2}=3$, and $\hat{l}^{2}+2 \hat{k} \hat{\ell}=5$ are not divisible by $\hat{n}=6$ (Proposition [3). Upside-down patterns do not exist because $(\hat{k}-\hat{\ell}) \operatorname{gcd}(\hat{k}, \hat{\ell})=1$ is divisible by $\operatorname{gcd}\left(\hat{k}^{2}+\hat{k} \hat{\ell}+\hat{\ell}^{2}, \hat{n}\right)=\operatorname{gcd}(7,6)=1$ (Proposition 4).

\footnotetext{
${ }^{9}$ The smallest $n$ for which the upside-down pattern exists is $n=21$ with $(k, \ell)=(9,1)$ and $(6,5)$. For $(k, \ell)=(9,1)$, $(\hat{k}-\hat{\ell}) \operatorname{gcd}(\hat{k}, \hat{\ell})=8$ is not divisible by $\operatorname{gcd}\left(\hat{k}^{2}+2 \hat{k} \hat{\ell}, \hat{n}\right)=\operatorname{gcd}(99,21)=3$ and $\operatorname{gcd}\left(\hat{k}^{2}+\hat{k} \hat{\ell}+\hat{\ell}^{2}, \hat{n}\right)=\operatorname{gcd}(91,21)=7$, thereby satisfying Proposition 4 For $(k, \ell)=(6,5),(\hat{k}-\hat{\ell}) \operatorname{gcd}(\hat{k}, \hat{\ell})=1$ is not divisible by $\operatorname{gcd}\left(\hat{k}^{2}+2 \hat{k} \hat{\ell}, \hat{n}\right)=\operatorname{gcd}(96,21)=3$ and by $\operatorname{gcd}\left(\hat{k}^{2}+\hat{k} \hat{\ell}+\hat{\ell}^{2}, \hat{n}\right)=\operatorname{gcd}(91,21)=7$.
} 
Table 1. Classification of bifurcating patterns for the $6 \times 6$ hexagonal lattice.

\begin{tabular}{lccc}
\hline$\mu$ & Hexagon (Type VM) & Racetrack & Stripe \\
\hline$(2)$ & $(w, 0)$ & $(-w, 0)$ & $\times$ \\
$(3)$ & $(w, w, w)$ & $(-w,-w,-w)$ & $(w, 0,0)$ \\
$(6 ; 1,0)$ & $(w, 0, w, 0, w, 0)$ & $(-w, 0,-w, 0,-w, 0)$ & $(w, 0,0,0,0,0)$ (Type I) \\
& & & $(0, w, 0,0,0,0)$ (Type II) \\
$(6 ; 2,0)$ & $(w, 0, w, 0, w, 0)$ & $(-w, 0,-w, 0,-w, 0)$ & $(w, 0,0,0,0,0)$ \\
$(6 ; 1,1)$ & $(w, 0, w, 0, w, 0)$ & $(-w, 0,-w, 0,-w, 0)$ & $(w, 0,0,0,0,0)$ (Type I) \\
$(12 ; 2,1)$ & $(w, 0, \ldots, w, 0)$ & $(-w, 0, \ldots,-w, 0)$ & $(0, w, 0,0,0,0)$ (Type II) \\
& & & $(w, 0,0, \ldots, 0)$ (Type I) \\
& & & $(0, w, 0, \ldots, 0)$ (Type II)
\end{tabular}

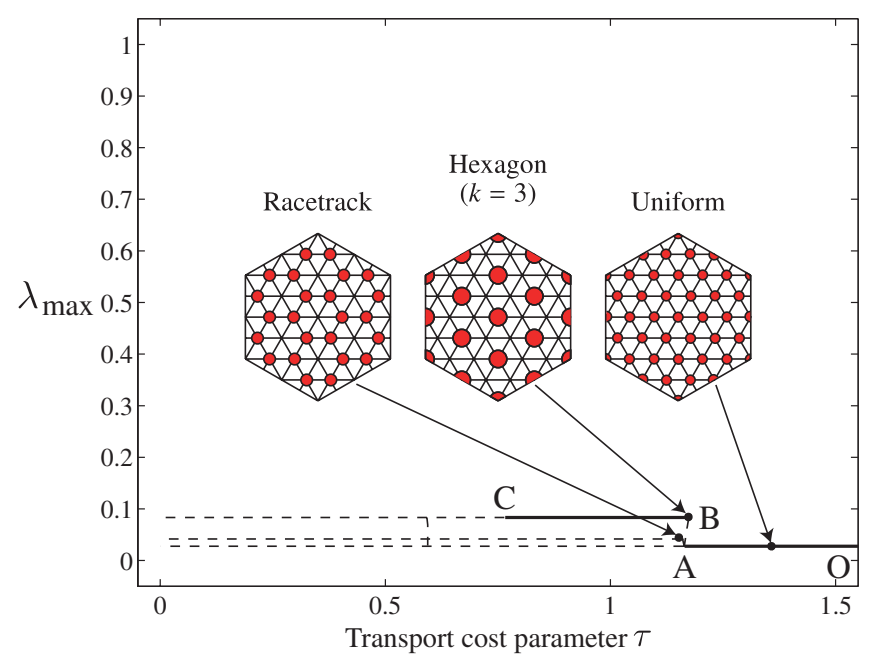

(a) $\mu=(2)$
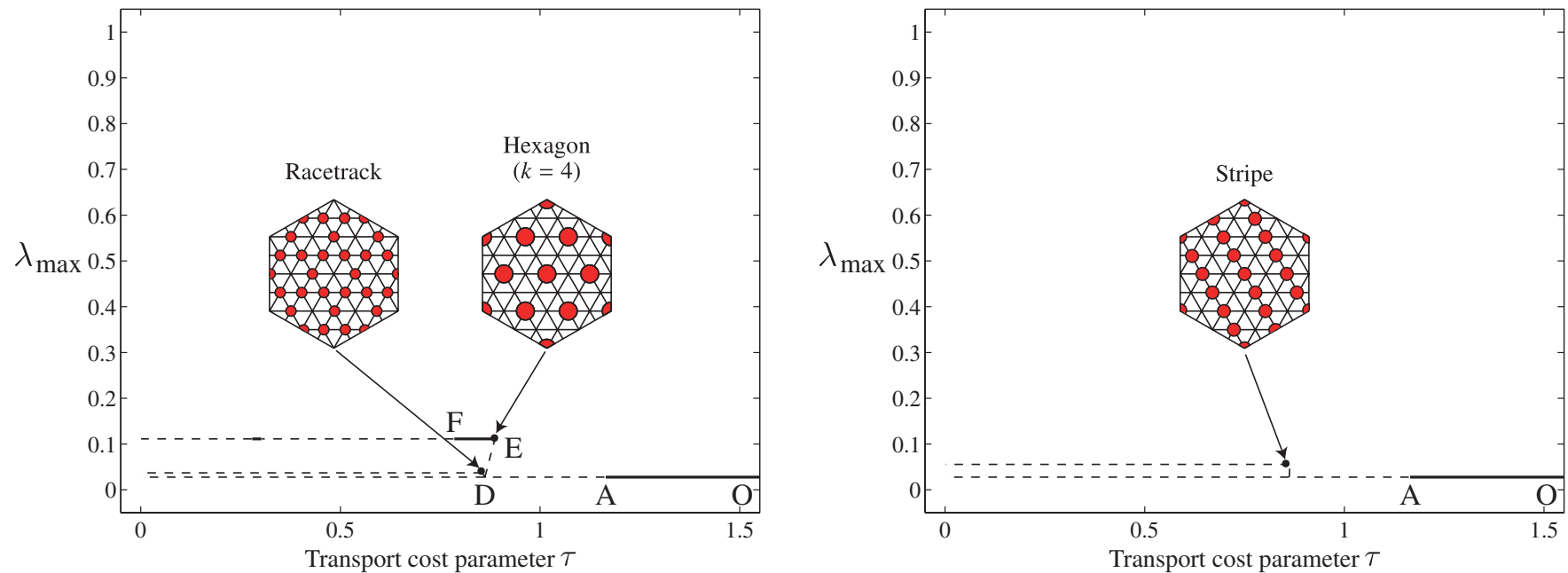

(b) $\mu=(3)$

Fig. 6. Bifurcating curves for $\mu=(2)$ and (3) (solid curves represent stable equilibria, dashed ones represent unstable ones). 


\subsection{A spatial economy model}

We consider the spatial economy model of Forslid and Ottaviano 2003. (see Ikeda et al., 2017) for multiplace version formulation of this model). In this model, there are two factors of production (skilled and unskilled labor), and two sectors (manufacture $\mathrm{M}$ and agriculture A). There, $H$ skilled and $L$ unskilled workers consume two final goods: manufacture-sector goods and agriculturesector goods. Workers supply one unit of each type of labor inelastically. Skilled workers are mobile across places, and the number of skilled workers in place $i$ is denoted by $\lambda_{i}$. Unskilled workers are immobile and equally distributed across all places with the unit density (i.e. $L=1 \times K$ ).

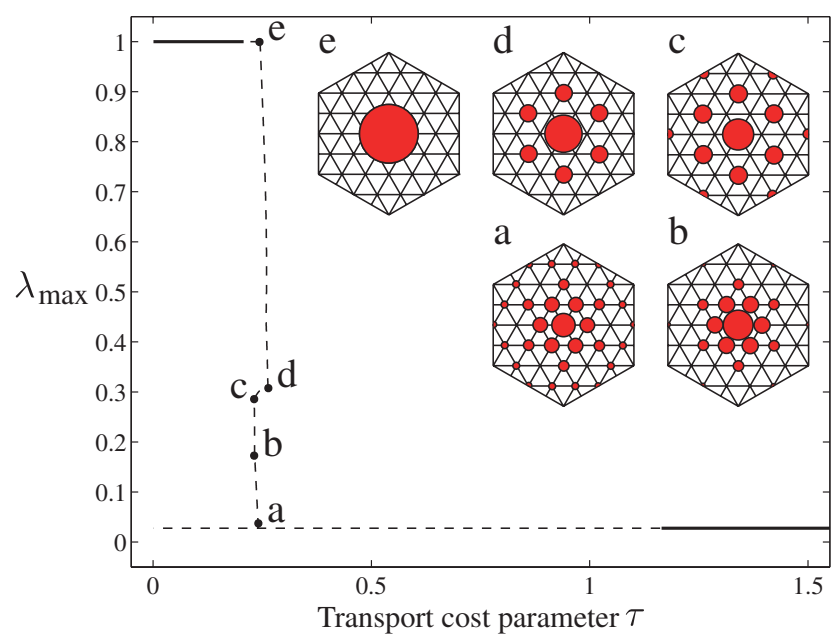

(a)

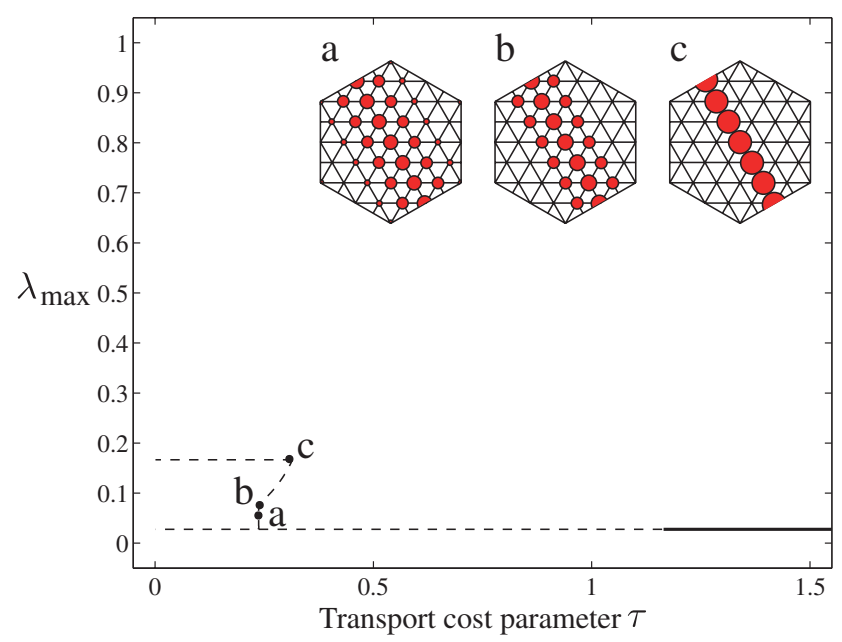

(c)
There are two parameters: $\mu$ is the constant expenditure share on industrial varieties, $\sigma$ expresses the constant elasticity of substitution between any two manufactured goods. Parameter values are chosen as $(\sigma, \mu)=(8.0,0.4)$. The distance unit is chosen as $d=1 / 6$.

\subsection{Numerical analysis on a spatial economy model}

The uniform state $\boldsymbol{\lambda}^{*}=\frac{1}{36}(1, \ldots, 1)^{\top}$ associated with $\mu=(1)$ is an equilibrium for any $\tau$ and an equilibrium curve is formed by $\left(\boldsymbol{\lambda}^{*}, \tau\right)$. The eigenanalysis was conducted on this equilibrium curve to find bifurcation points, corresponding to $\mu$ 's in (14).

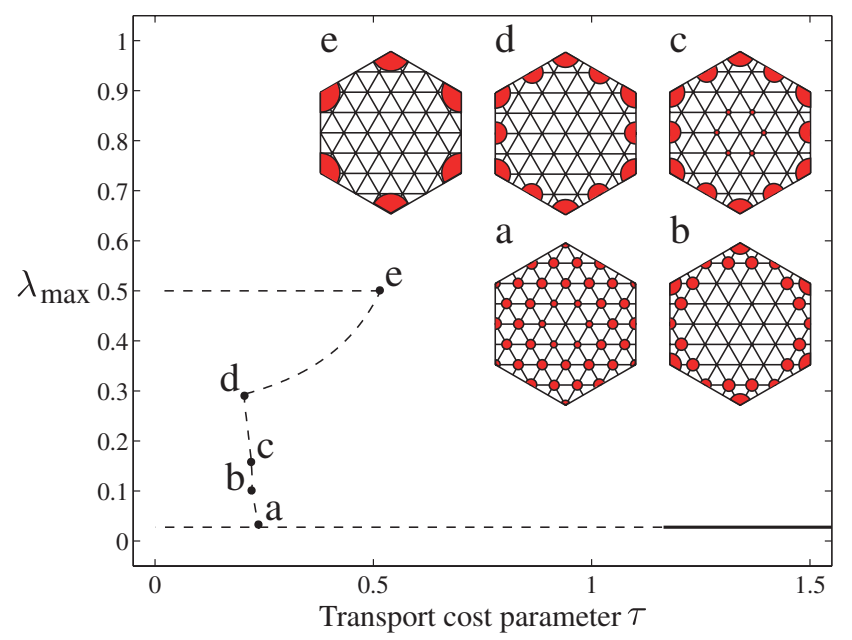

(b)

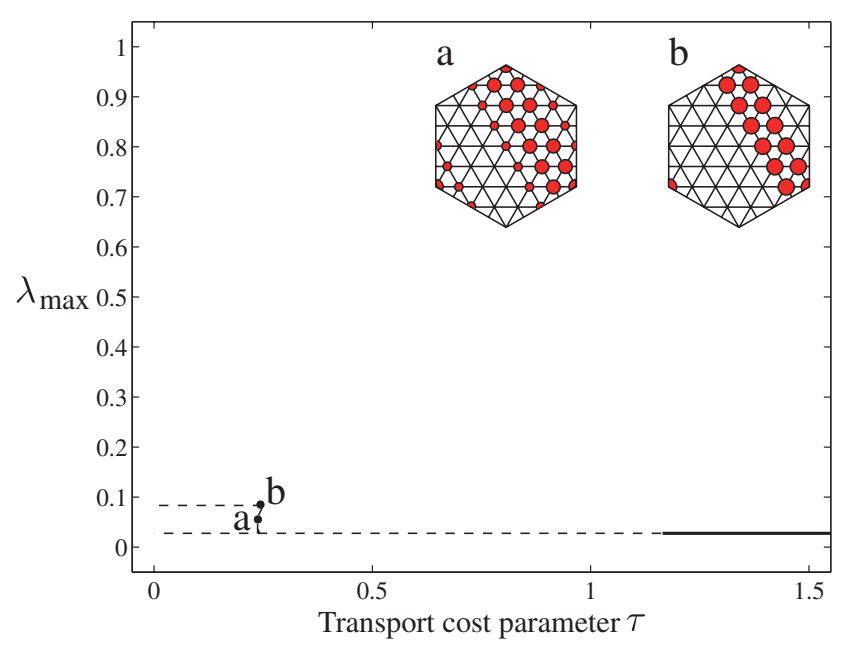

(d)

Fig. 7. Bifurcating curves for $\mu=(6 ; 1,0)$ (solid curves represent stable equilibria, dashed ones represent unstable ones). (a) Hexagon, (b) racetrack, (c) stripe (Type I) and (d) stripe (Type II). 


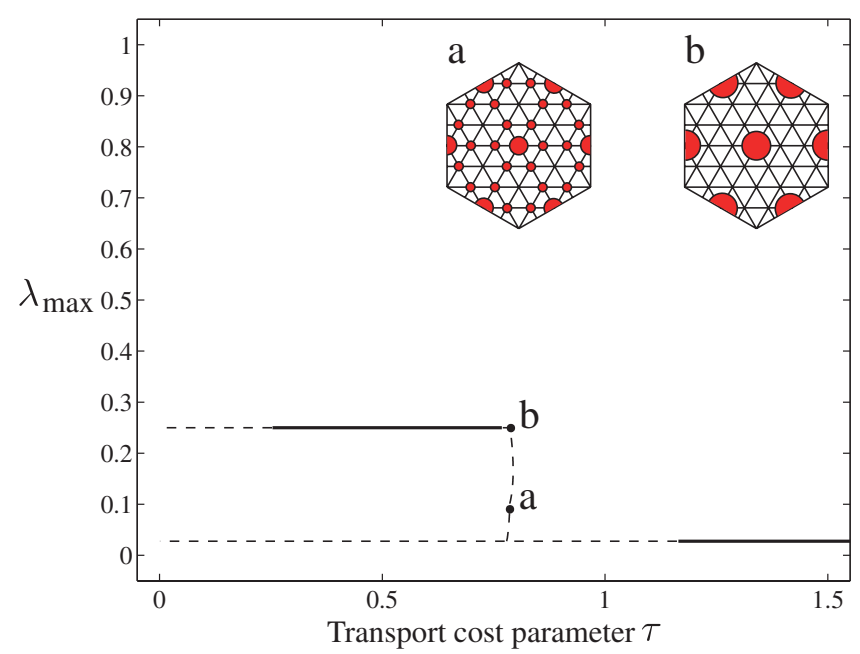

(a)

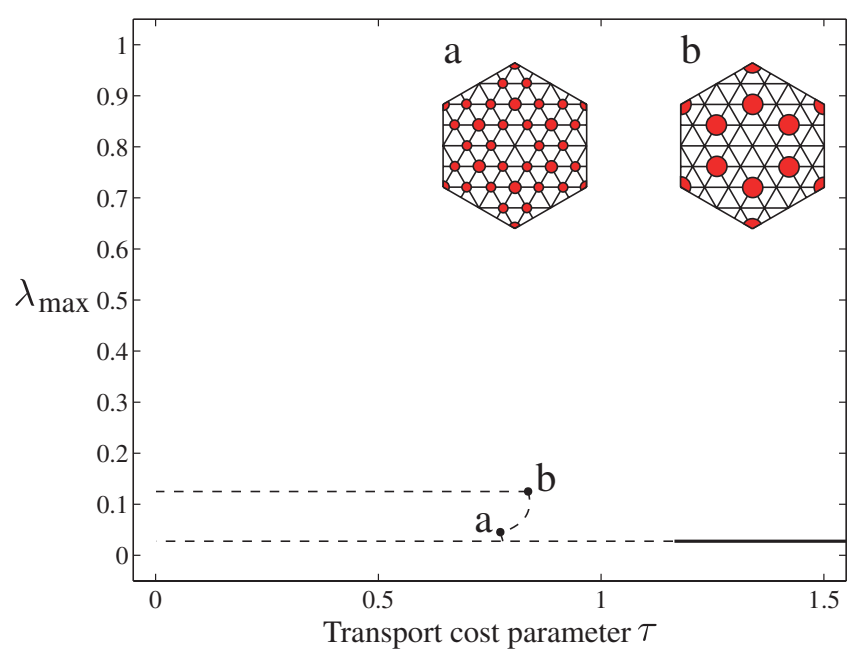

(b)

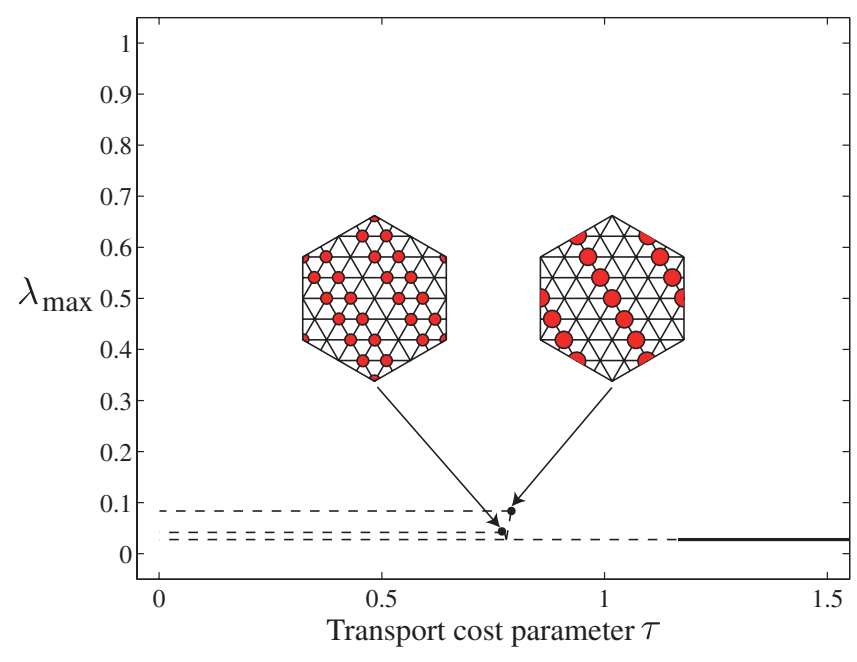

(c)

Fig. 8. Bifurcating curves for $\mu=(6 ; 2,0)$ (solid curves represent stable equilibria, dashed ones represent unstable ones). (a) Hexagon, (b) racetrack and (c) stripe (Type I). 


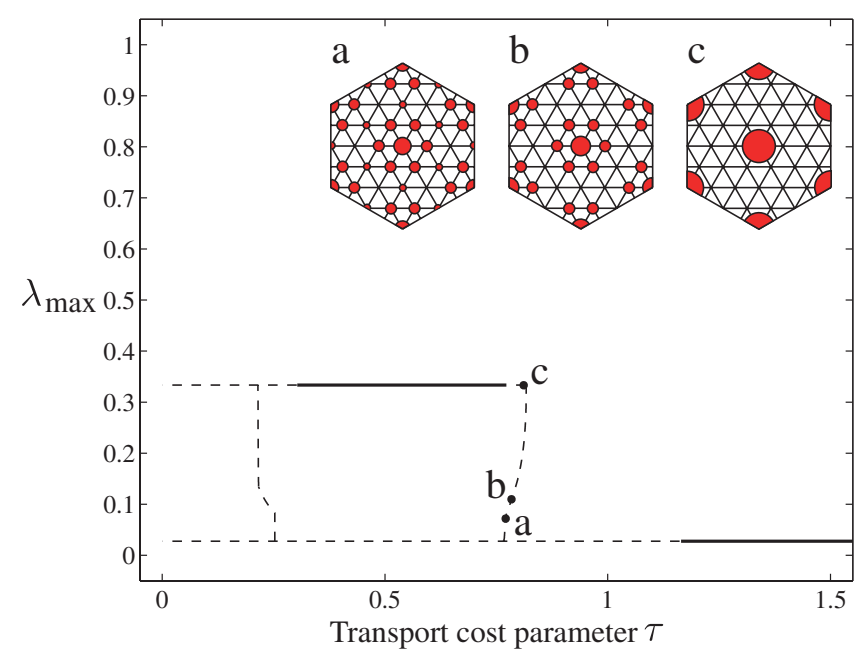

(a)

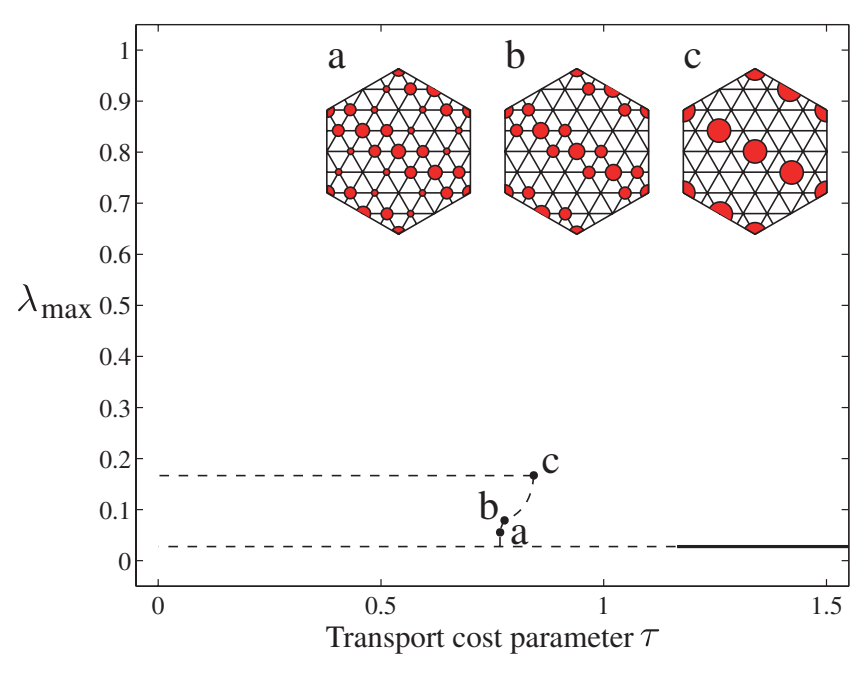

(c)

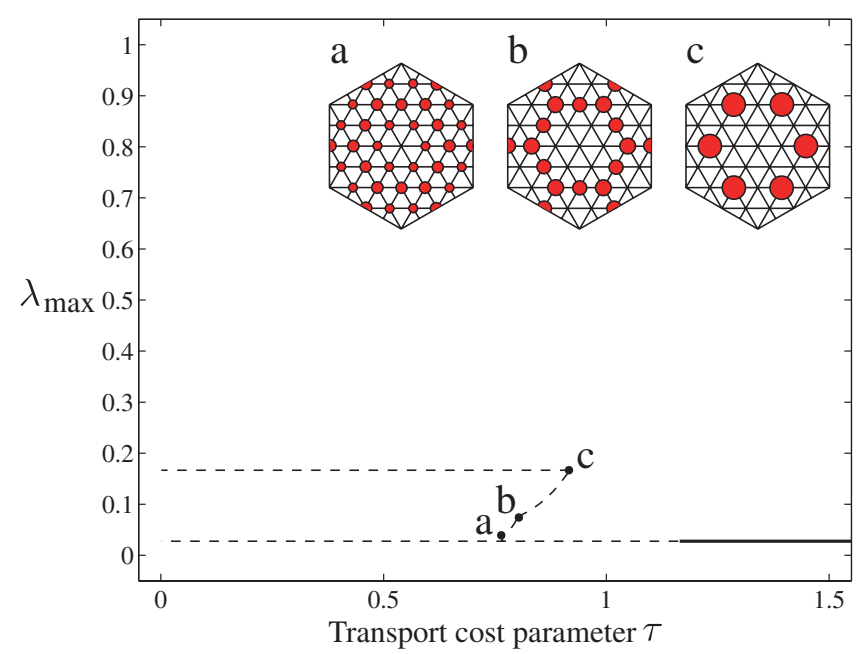

(b)

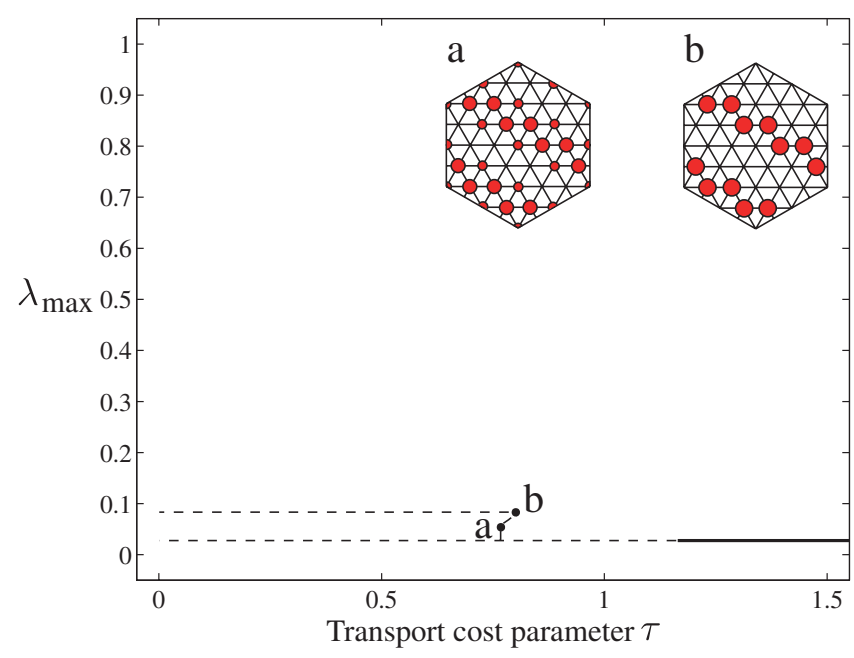

(d)

Fig. 9. Bifurcating curves for $\mu=(6 ; 1,1)$ (solid curves represent stable equilibria, dashed ones represent unstable ones). (a) Hexagon, (b) racetrack, (c) stripe (Type I) and (d) stripe (Type II). 


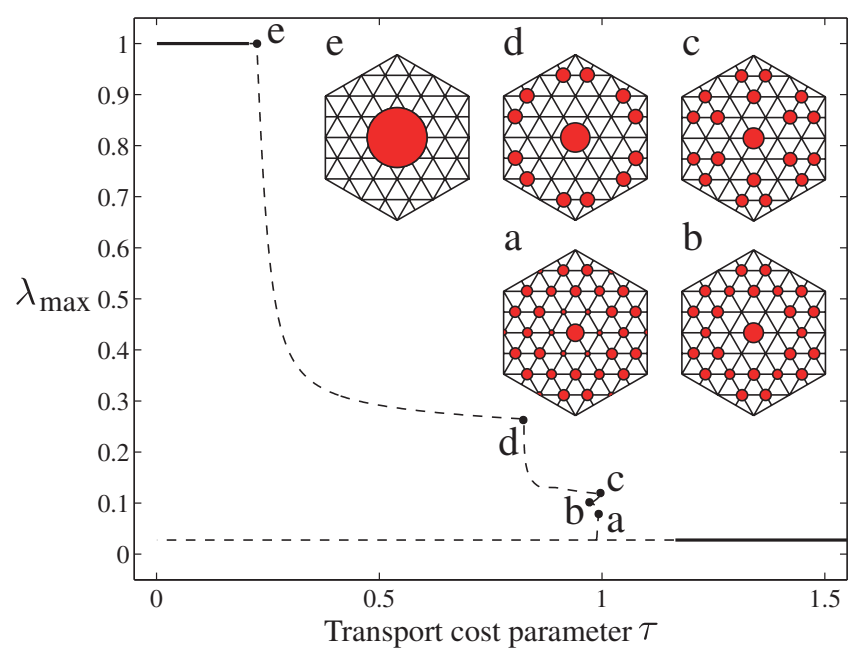

(a)

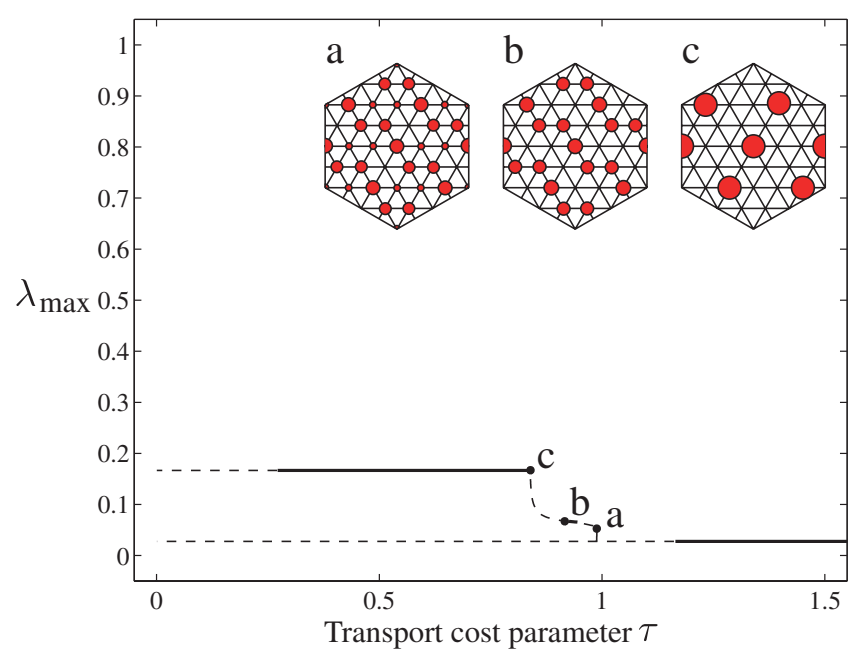

(c)

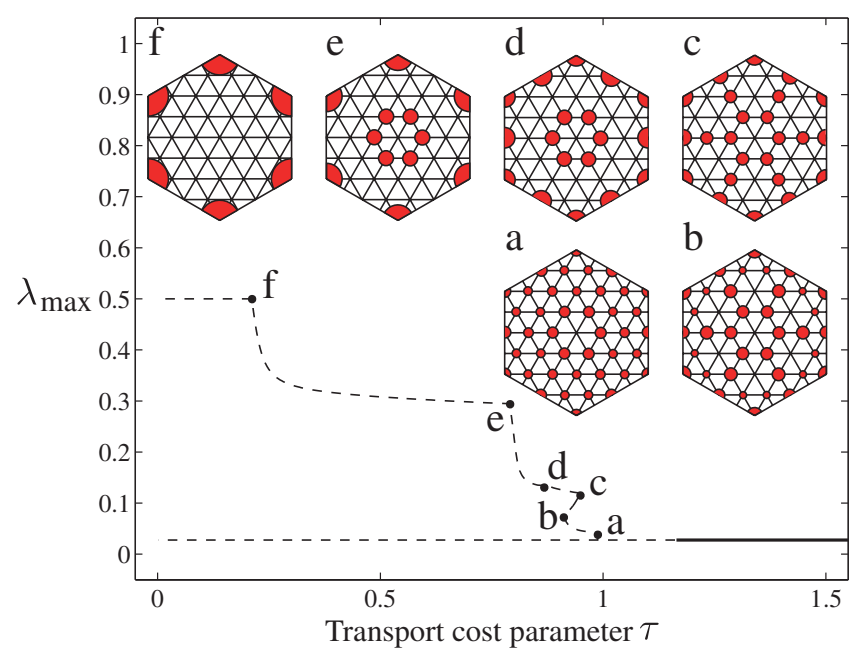

(b)

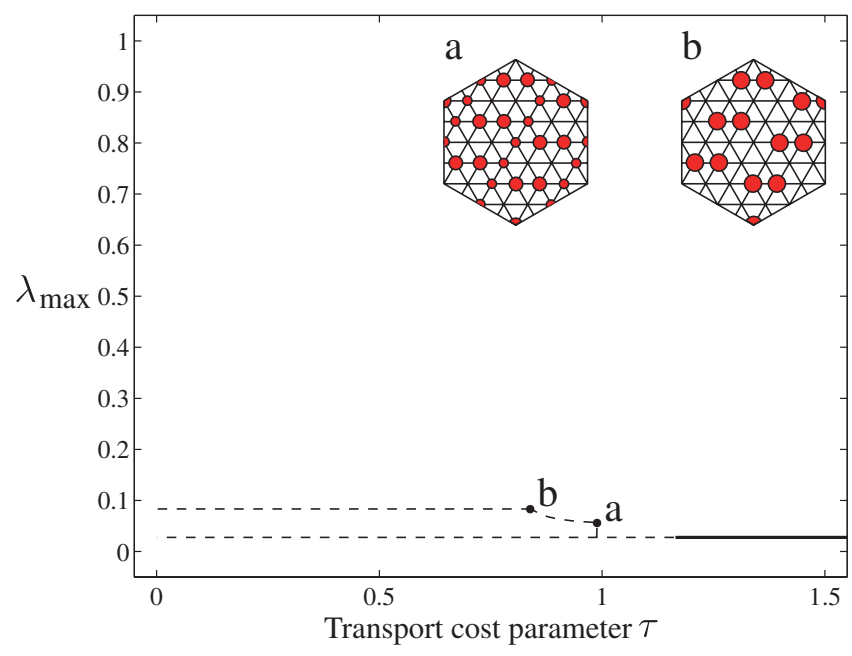

(d)

Fig. 10. Bifurcating curves for $\mu=(12 ; 2,1)$ (solid curves represent stable equilibria, dashed ones represent unstable ones). (a) Hexagon, (b) racetrack, (c) stripe (Type I) and (d) stripe (Type II).

Bifurcating equilibria branching from these bifurcation points had hexagonal, racetrack and stripe patterns as shown in Figs. 6610. The existence of these patterns is in agreement with the theoretical prediction in Sec. 4.1. The bifurcating solutions were all unstable just after bifurcation, as theoretically predicted in Sec. 4.2.

When the transport cost $\tau$ is decreased from a very large value, the bifurcation point $\mathrm{A}$ for $\mu=(2)$ is the first bifurcation point, at which the uniform state lost stability [Fig. 6(a)]. A bifurcating hexagonal pattern regained stability at point $\mathrm{B}$, whereas a bifurcating racetrack pattern remained unstable throughout. Such recovery of stability was observed for some other bifurcating solutions. All hexagonal patterns recovered stability, whereas all racetrack patterns remained unstable. The stripe pattern in Fig. 10(c) regained stability, whereas other stripe patterns were unstable. Thus hexagonal patterns that were advanced in favor of their superior economic properties in central place theory are superior also in stability, thereby supporting the foresight of this theory.

\section{Conclusions}

In the study of spatial economic agglomerations, a hexagonal lattice serves as one of the most realistic spatial platform. This paper has tried to exhaustively find bifurcating patterns of spatial economy 
models on this lattice by group-theoretic bifurcation analysis. Possible bifurcating patterns found herein were hexagonal, racetrack, stripe, and upside-down patterns. These hexagonal patterns are nothing but the ones which were predicted in central place theory. The stability of all these bifurcations are investigated and, in turn, to arrive at an amazing fact: all bifurcating solutions are unstable just after bifurcation for whatever models.

In the numerical analysis for a spatial economy model, all bifurcating equilibria were found to be unstable just after bifurcation but some equilibria recovered stability to engender stable agglomerations patterns. Hexagonal patterns that had been advanced in favor of their superior economic properties in central place theory were superior also in stability, thereby supporting the foresight of this theory. On the other hand, racetrack and stripe patterns were inferior in stability. Such features found herein are to be considered in future studies. This paper would contribute to the study of a two-dimensional spatial agglomeration in New Economic Geography through the introduction of a methodology in group-theoretic bifurcation theory Golubitsky et al., 1988].

\section{References}

Akamatsu, T., Takayama, Y. \& Ikeda, K. [2012] "Spatial discounting, Fourier, and racetrack economy: A recipe for the analysis of spatial agglomeration models," $J$. Econ. Dyn. Contr. 36, 1729-1759.

Banaszak, M., Dziecielski, M., Nijkamp, P. \& Ratajczak, W. [2015] "Self-organisation in spatial systems: From fractal chaos to regular patterns and vice versa," PLoS One 10, e0136248.

Batty, M. \& Longley, P. [1994] Fractal Cities (Academic Press, San Diego).

Bénard, H. [1900] "Les tourbillons cellulaires dans une nappe liquide," Rev. Gén. Sci. Pures Appl. 11, 12611271, 1309-1328.

Brakman, S., Garretsen, H., van Marrewijk, C. \& van den Berg, M. [1999] "The return of Zipf: Towards a further understanding of the rank-size distribution," J. Reg. Sci. 39, 183-213.

Buzano, E. \& Golubitsky, M. [1983] "Bifurcation on the hexagonal lattice and the planar Bénard problem," Phil. Trans. Roy. Soc. Lond. A 308, 617667.

Christaller, W. [1933] Die zentralen Orte in Süddeutschland (Gustav Fischer, Jena); English translation [1966] Central Places in Southern Germany (Prentice Hall, Englewood Cliffs).
Clarke, M. \& Wilson, A. G. [1983] "The dynamics of urban spatial structure: Progress and problems," $J$. Reg. Sci. 23, 1-18.

Dionne, B. \& Golubitsky, M. [1992] "Planforms in two and three dimensions," Zeit. Angew. Math. Phys. 43, $36-62$.

Dionne, B., Silber, M. \& Skeldon, A. C. [1997] "Stability results for steady, spatially, periodic planforms," Nonlinearity 10, 321-353.

Eaton, B. C. \& Lipsey, R. G. [1975] "The principle of minimum differentiation reconsidered: Some new developments in the theory of spatial competition," Rev. Econ. Stud. 42, 27-49.

Forslid, R. \& Ottaviano, G. I. P. [2003] "An analytically solvable core-periphery model," J. Econ. Geogr. 3, 229-340.

Fujita, M., Krugman, P. \& Mori, T. [1999a] "On the evolution of hierarchical urban systems," Eur. Econ. Rev. 43, 209-251.

Fujita, M., Krugman, P. \& Venables, A. J. [1999b] The Spatial Economy (MIT Press, Cambridge).

Golubitsky, M., Stewart, I. \& Schaeffer, D. G. [1988] Singularities and Groups in Bifurcation Theory, Vol. 2 (Springer, NY).

Golubitsky, M. \& Stewart, I. [2002] The Symmetry Perspective (Birkhäuser, Basel).

Ikeda, K. \& Murota, K. [2010] Imperfect Bifurcation in Structures and Materials, 2nd edition (Springer, NY).

Ikeda, K., Akamatsu, T. \& Kono, T. [2012a] "Spatial period-doubling agglomeration of a core-periphery model with a system of cities," J. Econ. Dyn. Contr. 36, 754-778.

Ikeda, K., Murota, K. \& Akamatsu, T. [2012b] "Selforganization of Lösch's hexagons in economic agglomeration for core-periphery models," Int. J. Bifurcation and Chaos 22, 1230026-1-29.

Ikeda, K. \& Murota, K. [2014] Bifurcation Theory for Hexagonal Agglomeration in Economic Geography (Springer, Tokyo).

Ikeda, K., Murota, K., Akamatsu, T., Kono, T. \& Takayama, Y. [2014] "Self-organization of hexagonal agglomeration patterns in new economic geography models," J. Econ. Behav. Organiz. 99, 32-52.

Ikeda, K., Murota, K. \& Takayama, Y. [2017] "Stable economic agglomeration patterns in two dimensions: Beyond the scope of central place theory," J. Reg. Sci. 57, 132-172.

Judd, S. L. \& Silber, M. [2000] "Simple and superlattice turning patterns in reaction-diffusion systems: Bifurcation, bistability, and parameter collapse," Physica $D$ 136, 45-65.

Kirchgässner, K. [1979] "Exotische Lösungen Bénardschen problems," Math. Meth. Appl. Sci. 1, 453-467.

Krugman, P. [1996] The Self-Organizing Economy (Blackwell, Malden). 
Lösch, A. [1954] The Economics of Location (Yale University Press, London).

Makse, H. A., Havlin, S. \& Stanley, H. E. [1995] "Modelling urban growth patterns," Nature 377, 608-612.

Munz, M. \& Weidlich, W. [1990] "Settlement formation, Part II: Numerical simulation," Ann. Reg. Sci. 24, 177-196.

Sanglier, M. \& Allen, P. [1989] "Evolutionary models of urban systems: An application to the Belgian provinces," Environ. Plan. A 21, 477-498.

Sattinger, D. H. [1978] "Group representation theory, bifurcation theory and pattern formation," J. Funct. Anal. 28, 58-101.

Stelder, D. [2005] "Where do cities form? A geographical agglomeration model for Europe," J. Reg. Sci. 45, 657-679.
Tabuchi, T. \& Thisse, J. F. [2011] "A new economic geography model of central places," J. Urban Econ. 69, 240-252.

Weidlich, W. \& Haag, G. [1987] "A dynamic phase transition model for spatial agglomeration processes," $J$. Reg. Sci. 27, 529-569.

\section{Appendices}

\section{Appendix A}

\section{Irreducible Representations}

Irreducible representations of the group $G=$ $\left\langle r, s, p_{1}, p_{2}\right\rangle$ are given by Ikeda and Murota [2014, Sec. 6.3] as

$$
\begin{aligned}
& T^{(2)}(r)=S, \quad T^{(2)}(s)=I, \\
& T^{(2)}\left(p_{1}\right)=T^{(2)}\left(p_{2}\right)=\left[\begin{array}{ll}
\cos \left(\frac{2 \pi}{3}\right) & -\sin \left(\frac{2 \pi}{3}\right) \\
\sin \left(\frac{2 \pi}{3}\right) & \cos \left(\frac{2 \pi}{3}\right)
\end{array}\right],
\end{aligned}
$$

$$
S=\operatorname{diag}(1,-1), \quad I=\operatorname{diag}(1,1),
$$$$
T^{(3)}(r)=\left[\begin{array}{lll} 
& & 1 \\
1 & & \\
& 1 &
\end{array}\right], \quad T^{(3)}(s)=\left[\begin{array}{rrr} 
& & 1 \\
& 1 & \\
1 & &
\end{array}\right], \quad\left\{\begin{array}{l}
T^{(3)}\left(p_{1}\right)=\operatorname{diag}(-1,1,-1), \\
T^{(3)}\left(p_{2}\right)=\operatorname{diag}(1,-1,-1),
\end{array}\right.
$$$$
T^{(6 ; k, 0)}(r)=\left[\begin{array}{cc} 
& S \\
S & \\
& S
\end{array}\right], \quad T^{(6 ; k, 0)}(s)=\left[\begin{array}{rr} 
& \\
& S \\
S &
\end{array}\right], \quad\left\{\begin{array}{l}
T^{(6 ; k, 0)}\left(p_{1}\right)=\operatorname{diag}\left(R^{k}, I, R^{-k}\right), \\
T^{(6 ; k, 0)}\left(p_{2}\right)=\operatorname{diag}\left(I, R^{-k}, R^{k}\right),
\end{array}\right.
$$$$
T^{(6 ; k, k)}(r)=\left[\begin{array}{ccc} 
& & S \\
S & & \\
& S &
\end{array}\right], \quad T^{(6 ; k, k)}(s)=\left[\begin{array}{ccc}
I & \\
I & & \\
& & I
\end{array}\right], \quad\left\{\begin{array}{l}
T^{(6 ; k, k)}\left(p_{1}\right)=\operatorname{diag}\left(R^{k}, R^{k}, R^{-2 k}\right), \\
T^{(6 ; k, k)}\left(p_{2}\right)=\operatorname{diag}\left(R^{k}, R^{-2 k}, R^{k}\right),
\end{array}\right.
$$

$$
R=\left[\begin{array}{cc}
\cos \left(\frac{2 \pi}{n}\right) & -\sin \left(\frac{2 \pi}{n}\right) \\
\sin \left(\frac{2 \pi}{n}\right) & \cos \left(\frac{2 \pi}{n}\right)
\end{array}\right], \quad T^{(12 ; k, \ell)}(r)=\left[\begin{array}{lll|l}
S & & \\
& S & & \\
\hline & & & \\
& & S & S
\end{array}\right],
$$




$$
T^{(12 ; k, \ell)}(s)=\left[\begin{array}{ll|lll}
I & & \\
& & I & \\
& & & I \\
& & & & \\
\hline I & & & &
\end{array}\right], \quad\left\{\begin{array}{l}
T^{(12 ; k, \ell)}\left(p_{1}\right)=\operatorname{diag}\left(R^{k}, R^{\ell}, R^{-k-\ell}, R^{k}, R^{\ell}, R^{-k-\ell}\right), \\
T^{(12 ; k, \ell)}\left(p_{2}\right)=\operatorname{diag}\left(R^{\ell}, R^{-k-\ell}, R^{k}, R^{-k-\ell}, R^{k}, R^{\ell}\right) .
\end{array}\right.
$$

\section{Appendix B}

\section{Stability of Bifurcating Solutions}

Stability of bifurcating solutions is investigated.

\section{B.1. Double bifurcation point}

For $\mu=(2)$, the bifurcation equation in the complex variable $z=w_{1}+\mathrm{i} w_{2}$ was evaluated to [Ikeda \& Murota, 2014, Sec. 9.3.2]

$$
\begin{aligned}
F(z, \bar{z}, \tilde{\tau}) & =\sum_{a=0}^{\infty} A_{a+1, a}(\tilde{\tau}) z^{a+1} \bar{z}^{a}+\sum_{p=1}^{\infty} \sum_{a=0}^{\infty}\left[A_{a+1+3 p, a}(\tilde{\tau}) z^{a+1+3 p} \bar{z}^{a}+A_{a, a-1+3 p}(\tilde{\tau}) z^{a} \bar{z}^{a-1+3 p}\right] \\
& \approx A_{10}^{\prime}(0) \tilde{\tau} z+A_{02}(0) \bar{z}^{2}
\end{aligned}
$$

where $A_{a+1,1}(\tilde{\tau}), A_{a+1+3 p, a}(\tilde{\tau})$, and $A_{a, a-1+3 p}(\tilde{\tau})$ are real and generically nonzero. The Jacobian matrix of the bifurcation equation is evaluated to

$$
\tilde{J}\left(w_{1}, w_{2}, \tilde{\tau}\right)=\left(\begin{array}{cc}
\frac{\partial \operatorname{Re} F}{\partial w_{1}} & \frac{\partial \operatorname{Re} F}{\partial w_{2}} \\
\frac{\partial \operatorname{Im} F}{\partial w_{1}} & \frac{\partial \operatorname{Im} F}{\partial w_{2}}
\end{array}\right) \approx\left(\begin{array}{cc}
A_{01}^{\prime}(0) \tilde{\tau}+2 A_{02}(0) w_{1} & -2 A_{02}(0) w_{2} \\
-2 A_{02}(0) w_{2} & A_{10}^{\prime}(0) \tilde{\tau}-2 A_{02}(0) w_{1}
\end{array}\right)
$$

The bifurcation equation $F=0$ has a bifurcating solution $z=w$, i.e. $\left(w_{1}, w_{2}\right)=(w, 0)(w \in \mathbb{R})$ and its solution curve is given by

$$
\tilde{\tau}=\tilde{\tau}_{1} \approx-\frac{A_{02}(0)}{A_{10}^{\prime}(0)} w .
$$

The substitution of $\left(w_{1}, w_{2}, \tilde{\tau}\right)=\left(w, 0, \tilde{\tau}_{1}\right)$ into (B.1) leads to $\tilde{J}\left(w, 0, \tilde{\tau}_{1}\right) \approx A_{02}(0) w \operatorname{diag}(1,-3)$. This Jacobian matrix has two eigenvalues $\lambda_{1} \approx$ $A_{02}(0) w$ and $\lambda_{2} \approx-3 A_{02}(0) w$ with opposite signs. The bifurcating solution, accordingly, is asymptotically unstable for any $A_{02}(0) w$. The racetrack pattern can be shown to be unstable by replacing $w$ by $-w$ in the discussion above.

\section{B.2. Triple bifurcation point (stripe pattern)}

The Jacobian matrix $J\left(w_{1}, w_{2}, w_{3}, \tilde{\tau}\right)$ for the set of bifurcation equations $\tilde{\mathbf{F}}$ in (8)-(10) is evaluated to

$$
\begin{aligned}
& \tilde{J}\left(w_{1}, w_{2}, w_{3}, \tilde{\tau}\right) \\
& \quad \approx\left(\begin{array}{ccc}
A_{010}^{\prime}(0) \tilde{\tau} & A_{101}(0) w_{3} & A_{101}(0) w_{2} \\
A_{101}(0) w_{3} & A_{010}^{\prime}(0) \tilde{\tau} & A_{101}(0) w_{1} \\
A_{101}(0) w_{2} & A_{101}(0) w_{1} & A_{010}^{\prime}(0) \tilde{\tau}
\end{array}\right) .
\end{aligned}
$$

For the stripe pattern with $\mathbf{w}_{\text {stripe }}=(w, 0,0)$ for $\mu=(3)$, we have

$$
\begin{aligned}
& \tilde{F}_{1}\left(\mathbf{w}_{\text {stripe }}, \tilde{\tau}\right)=A_{010}^{\prime}(0) \tilde{\tau} w+\sum_{b=0}^{\infty} A_{0,2 b+1,0}(\tilde{\tau}) w^{2 b+1}, \\
& \tilde{F}_{2}\left(\mathbf{w}_{\text {stripe }}, \tilde{\tau}\right)=\tilde{F}_{3}\left(\mathbf{w}_{\text {stripe }}, \tilde{\tau}\right)=0 .
\end{aligned}
$$

Thus we have a bifurcating solution

$$
\tilde{\tau}=\tilde{\tau}_{2} \approx-\frac{A_{030}(0) w^{2}}{A_{010}^{\prime}(0)}
$$


and the Jacobian matrix

$$
\tilde{J}\left(\mathbf{w}_{\text {stripe }}, \tilde{\tau}_{2}\right) \approx A_{101}(0) w\left(\begin{array}{ccc}
0 & 0 & 0 \\
0 & 0 & 1 \\
0 & 1 & 0
\end{array}\right),
$$

in which the diagonal terms have been omitted because they are higher order terms than $A_{101}(0) w$. This Jacobian matrix has eigenvalues $\lambda_{1} \approx$ $-A_{101}(0) w$ and $\lambda_{2} \approx A_{101}(0) w$ with opposite signs; accordingly, the bifurcating stripe pattern is asymptotically unstable for any $A_{101}(0) w$.

\section{B.3. Sextuple bifurcation point}

The bifurcation equation for $\mu=(6 ; k, \ell)(\ell=0, k)$ can be expressed as a three-dimensional complexvalued equation in complex variables $z_{1}, z_{2}, z_{3}\left(z_{i}=\right.$ $\left.w_{2 i-1}+w_{2 i} \mid i=1,2,3\right)$ as

$$
F_{i}\left(z_{1}, z_{2}, z_{3}, \bar{z}_{1}, \bar{z}_{2}, \bar{z}_{3}, \tilde{\tau}\right)=0, \quad i=1,2,3 \text {. }
$$

We expand $F_{1}$ as

$$
\begin{aligned}
& F_{1}\left(z_{1}, z_{2}, z_{3}, \bar{z}_{1}, \bar{z}_{2}, \bar{z}_{3}, \tilde{\tau}\right) \\
& \quad=\sum_{a=0} \sum_{b=0} \sum_{c=0} \sum_{d=0} \sum_{e=0} \sum_{g=0} A_{a b c d e g}(\tilde{\tau}) z_{1}^{a} z_{2}^{b} z_{3}^{c} \bar{z}_{1}^{d} \bar{z}_{2}^{e} \bar{z}_{3}^{g} .
\end{aligned}
$$

Since $\left(z_{1}, z_{2}, z_{3}, \bar{z}_{1}, \bar{z}_{2}, \bar{z}_{3}, \tilde{\tau}\right)=(0, \ldots, 0,0)$ corresponds to the critical point,

$$
\begin{gathered}
A_{000000}(0)=0 \\
A_{100000}(0)=A_{010000}(0)=\cdots=A_{000001}(0)=0
\end{gathered}
$$

are satisfied. Therefore, we have $A_{100000}(\tilde{\tau}) \approx A \tilde{\tau}$ for $A=A_{100000}^{\prime}(0)$. We omit $\bar{z}_{1}, \bar{z}_{2}, \bar{z}_{3}$, and $\tilde{\tau}$ in the arguments of $F_{i}$ in the subsequent derivation.

The equivariance with respect to $r$ led to $A_{\text {abcdeg }} \in \mathbb{R}$ and

$$
\begin{aligned}
& F_{2}\left(z_{1}, z_{2}, z_{3}\right)=F_{1}\left(z_{2}, z_{3}, z_{1}\right), \\
& F_{3}\left(z_{1}, z_{2}, z_{3}\right)=F_{1}\left(z_{3}, z_{1}, z_{2}\right)
\end{aligned}
$$

Ikeda \& Murota, 2014, Sec. 9.5.2]. The equivariance to $p_{1}$ and to $p_{2}$ led for $\mu=(6 ; k, 0)$ to

$$
\begin{aligned}
a-d-c+g-1 & \equiv 0 \quad \bmod \hat{n}, \\
-b+e+c-g & \equiv 0 \quad \bmod \hat{n}
\end{aligned}
$$

$\left(\hat{n}=\frac{n}{\operatorname{gcd}(k, n)}\right)$ and for $\mu=(6 ; k, k)$ to

$$
\begin{aligned}
a-d-c+g-1 & \equiv 0 \quad \bmod \tilde{n}, \\
-b+e+c-g & \equiv 0 \quad \bmod \tilde{n},
\end{aligned}
$$

where $\tilde{n}=\hat{n} / 3$ if $\hat{n} \in 3 \mathbb{Z}$ and $\tilde{n}=\hat{n}$ if $\hat{n} \notin 3 \mathbb{Z}$ ( $\mathbb{Z}$ is the set of integers).

A system of bifurcation equations $F_{1}=F_{2}=$ $F_{3}=0$ has the following bifurcating solutions:

(i) $\mathbf{w}_{\text {hexa }}=(w, 0, w, 0, w, 0)$ : hexagonal pattern.

(ii) $\mathbf{w}_{\text {racetrack }}=(-w, 0,-w, 0,-w, 0)$ : racetrack pattern.

(iii) $\mathbf{w}_{\text {stripeI }}=(w, 0,0,0,0,0)$ : stripe pattern I.

(iv) $\mathbf{w}_{\text {stripeII }}=(0, w, 0,0,0,0)$ : stripe pattern II.

Here the first three solutions were shown to exist Ikeda \& Murota, 2014, Sec. 9.5.2], whereas the last one has been newly advanced herein (Sec. B.3.3).

We can express $F_{1}$ asymptotically as

$$
F_{1}\left(z_{1}, z_{2}, z_{3}\right) \approx \begin{cases}A_{100000}(\tilde{\tau}) z_{1}+A_{000011}(\tilde{\tau}) \bar{z}_{2} \bar{z}_{3}+A_{000200}(\tilde{\tau}) \bar{z}_{1}^{2} & \text { for } \hat{n}=3(\mu=(6 ; k, 0)), \\ A_{100000}(\tilde{\tau}) z_{1}+A_{000011}(\tilde{\tau}) \bar{z}_{2} \bar{z}_{3} & \text { for } \hat{n} \neq 3\end{cases}
$$

and $F_{2}$ and $F_{3}$ can be obtained from (B.6) by permuting $z_{1}, z_{2}$, and $z_{3}$ in $F_{1}$. We hereafter set $a_{1} \equiv$ $A_{100000}^{\prime}(0), a_{2} \equiv A_{000011}(0)$, and $a_{3} \equiv A_{000200}(0)\left(A_{100000}(\tilde{\tau}) \approx A_{100000}(0)+A_{100000}^{\prime}(0) \tilde{\tau}=A_{100000}^{\prime}(0) \tilde{\tau}\right)$. Then we have

$$
\tilde{J}(\mathbf{w}, \tilde{\tau}) \approx\left(\begin{array}{cccccc}
a_{1} \tilde{\tau}+2 a_{3} w_{1} & -2 a_{3} w_{2} & a_{2} w_{5} & -a_{2} w_{6} & a_{2} w_{3} & -a_{2} w_{4} \\
-2 a_{3} w_{2} & a_{1} \tilde{\tau}-2 a_{3} w_{1} & -a_{2} w_{6} & -a_{2} w_{5} & -a_{2} w_{4} & -a_{2} w_{3} \\
a_{2} w_{5} & -a_{2} w_{6} & a_{1} \tilde{\tau}+2 a_{3} w_{3} & -2 a_{3} w_{4} & a_{2} w_{1} & -a_{2} w_{2} \\
-a_{2} w_{6} & -a_{2} w_{5} & -2 a_{3} w_{4} & a_{1} \tilde{\tau}-2 a_{3} w_{3} & -a_{2} w_{2} & -a_{2} w_{1} \\
a_{2} w_{3} & -a_{2} w_{4} & a_{2} w_{1} & -a_{2} w_{2} & a_{1} \tilde{\tau}+2 a_{3} w_{5} & -2 a_{3} w_{6} \\
-a_{2} w_{4} & -a_{2} w_{3} & -a_{2} w_{2} & -a_{2} w_{1} & -2 a_{3} w_{6} & a_{1} \tilde{\tau}-2 a_{3} w_{5}
\end{array}\right)
$$$$
\text { for } \hat{n}=3 \text {, }
$$ 


$$
\tilde{J}(\mathbf{w}, \tilde{\tau}) \approx\left(\begin{array}{cccccc}
a_{1} \tilde{\tau} & 0 & a_{2} w_{5} & -a_{2} w_{6} & a_{2} w_{3} & -a_{2} w_{4} \\
0 & a_{1} \tilde{\tau} & -a_{2} w_{6} & -a_{2} w_{5} & -a_{2} w_{4} & -a_{2} w_{3} \\
a_{2} w_{5} & -a_{2} w_{6} & a_{1} \tilde{\tau} & 0 & a_{2} w_{1} & -a_{2} w_{2} \\
-a_{2} w_{6} & -a_{2} w_{5} & 0 & a_{1} \tilde{\tau} & -a_{2} w_{2} & -a_{2} w_{1} \\
a_{2} w_{3} & -a_{2} w_{4} & a_{2} w_{1} & -a_{2} w_{2} & a_{1} \tilde{\tau} & 0 \\
-a_{2} w_{4} & -a_{2} w_{3} & -a_{2} w_{2} & -a_{2} w_{1} & 0 & a_{1} \tilde{\tau}
\end{array}\right) \quad \text { for } \hat{n} \neq 3
$$

\section{B.3.1. Hexagonal and racetrack patterns}

The bifurcating solution curve of the hexagonal pattern is given by

$$
\begin{cases}a_{1} \tilde{\tau}_{1}+\left(a_{2}+a_{3}\right) w \approx 0 & \text { for } \hat{n}=3, \\ a_{1} \tilde{\tau}_{2}+a_{2} w \approx 0 & \text { for } \hat{n} \neq 3 .\end{cases}
$$

For $\hat{n}=3$, the use of $\mathbf{w}_{\text {hexa }}=(w, 0, w, 0, w, 0)$ and $a_{1} \tilde{\tau}_{1} \approx-\left(a_{2}+a_{3}\right) w$ in (‥9) leads to

$$
\tilde{J}\left(\mathbf{w}_{\text {hexa }}, \tilde{\tau}_{1}\right) \approx a_{2} w\left(\begin{array}{cccccc}
-1+x & 0 & 1 & 0 & 1 & 0 \\
0 & -1-3 x & 0 & -1 & 0 & -1 \\
1 & 0 & -1+x & 0 & 1 & 0 \\
0 & -1 & 0 & -1-3 x & 0 & -1 \\
1 & 0 & 1 & 0 & -1+x & 0 \\
0 & -1 & 0 & -1 & 0 & -1-3 x
\end{array}\right)
$$

with $x=a_{3} / a_{2}$. The eigenvalues of this matrix are

$$
\left\{\begin{array}{l}
\lambda_{1} \approx-3\left(a_{2}+a_{3}\right) w, \quad \lambda_{2} \approx\left(a_{2}+a_{3}\right) w \\
\lambda_{3} \approx-\left(2 a_{2}-a_{3}\right) w, \quad \lambda_{4} \approx-3 a_{3} w \quad \text { (repeated twice) } .
\end{array}\right.
$$

Since the eigenvalues $\lambda_{1}$ and $\lambda_{2}$ have opposite signs, the associated bifurcating solution is always asymptotically unstable.

For $\hat{n} \neq 3$, the use of $\mathbf{w}_{\text {hexa }}=(w, 0, w, 0, w, 0)$ and $a_{1} \tilde{\tau}_{2} \approx-a_{2} w$ in (B.10) gives

$$
\tilde{J}\left(\mathbf{w}_{\text {hexa }}, \tilde{\tau}_{2}\right) \approx a_{2} w A_{1} \quad \text { with } A_{1}=\left(\begin{array}{ccc}
-I & S & S \\
S & -I & S \\
S & S & -I
\end{array}\right), \quad I=\left(\begin{array}{ll}
1 & 0 \\
0 & 1
\end{array}\right), \quad S=\left(\begin{array}{rr}
1 & 0 \\
0 & -1
\end{array}\right) .
$$

The eigenvalues of the matrix $\tilde{J}\left(\mathbf{w}_{\text {hexa }}, \tilde{\tau}_{2}\right)$ are $\lambda_{1} \approx-3 a_{2} w, \lambda_{2} \approx a_{2} w, \lambda_{3} \approx-2 a_{2} w$ (repeated twice), and $\lambda_{4} \approx 0$ (repeated twice). Since $\lambda_{1}$ and $\lambda_{2}$ have opposite signs, the associated bifurcating hexagonal solution is always asymptotically unstable. The bifurcating racetrack solution can be shown as also asymptotically unstable by replacing $w$ with $-w$ in the discussion above.

\section{B.3.2. Type I stripe pattern}

The bifurcating solution curve of the Type I stripe pattern is given by

$$
\begin{cases}a_{1} \tilde{\tau}_{3}+a_{3} w \approx 0 & \text { for } \hat{n}=3 \\ a_{1} \tilde{\tau}_{4}+a_{4} w^{2} \approx 0 & \text { for } \hat{n} \neq 3\end{cases}
$$


with $a_{4}=A_{000300}(0)+A_{200100}(0)$ for $\hat{n}=4$ and $a_{4}=A_{200100}(0)$ for $\hat{n} \geq 5$.

For $\hat{n}=3$, the use of $\mathbf{w}_{\text {stripeI }}=(w, 0,0,0,0,0)$ and $a_{1} \tilde{\tau}_{3} \approx-a_{3} w$ in (B.9) yields

$$
\approx a_{3} w\left(\begin{array}{ccc}
D & O & O \\
O & -I & \frac{a_{2}}{a_{3}} S \\
O & \frac{a_{2}}{a_{3}} S & -I
\end{array}\right) \text { with } D=\left(\begin{array}{rr}
1 & 0 \\
0 & -3
\end{array}\right) .
$$

The eigenvalues of this Jacobian matrix are $\lambda_{1} \approx$ $-3 a_{3} w, \lambda_{2} \approx a_{3} w, \lambda_{3} \approx-\left(a_{2}+a_{3}\right) w$ (repeated twice), and $\lambda_{4} \approx\left(a_{2}-a_{3}\right) w$ (repeated twice). Since the eigenvalues $\lambda_{1}$ and $\lambda_{2}$ have opposite signs regardless of the sign of $a_{3} w$, the associated bifurcating solution is always asymptotically unstable.

For $\hat{n} \neq 3$, the use of $\mathbf{w}_{\text {stripeI }}=(w, 0,0,0,0,0)$ and $a_{1} \tilde{\tau}_{4} \approx-a_{4} w^{2}$ [cf. (B.12)] in (B.10) leads to

$$
\begin{aligned}
& \tilde{J}\left(\mathbf{w}_{\text {stripeI }}, \tilde{\tau}_{4}\right) \\
& \approx a_{2} w A_{2} \quad \text { with } A_{2}=\left(\begin{array}{ccc}
O & O & O \\
O & O & S \\
O & S & O
\end{array}\right)
\end{aligned}
$$

and the diagonal terms $-\frac{a_{4} w^{2}}{a_{2}}$ have been omitted because they are higher order terms than $a_{2} w$. The eigenvalues of the matrix $\tilde{J}\left(\mathbf{w}_{\text {stripeI }}, \tilde{\tau}_{4}\right)$ are $\lambda_{1} \approx-a_{2} w, \lambda_{2} \approx a_{2} w$, and $\lambda_{3} \approx 0$ (repeated twice). Since the eigenvalues $\lambda_{1}$ and $\lambda_{2}$ have opposite signs, the associated bifurcating solution is always asymptotically unstable.

\section{B.3.3. Type II stripe pattern}

Let us search for a solution of the form $\mathbf{w}_{\text {stripeII }}=$ $(0, w, 0,0,0,0) \quad\left(z_{1}, z_{2}, z_{3}\right)=(\mathrm{i} w, 0,0)$. For $\mu=$ $(6 ; k, 0)$, from (B.7), we have $a=d+1+p \hat{n}(p \in \mathbb{Z})$. Such solution satisfies $F_{2}=F_{3}=0$, whereas $F_{1}$ reduces to

$$
\frac{F_{1}(\mathrm{i} w, 0,0,-\mathrm{i} w, 0,0)}{\mathrm{i}}=\sum_{d+1+p \hat{n} \geq 0, d \geq 0} A_{d+1+p \hat{n}, 00 d 00}(\tilde{\tau})(-1)^{d} \mathrm{i}^{2 d+p \hat{n}} w^{1+2 d+p \hat{n}} .
$$

Then we see that $F_{1}(\mathrm{i} w, 0,0,-\mathrm{i} w, 0,0)=0$ has a solution if $\hat{n}$ is even, for which $\mathrm{i}^{2 d+p \hat{n}}=(-1)^{d+p \hat{n} / 2}$ is always real. Similarly for $\mu=(6 ; k, k)$, the solution exists if $\tilde{n}$ is even.

The use of $\mathbf{w}_{\text {stripeII }}=(0, w, 0,0,0,0)$ and its solution curve $a_{1} \tilde{\tau}_{4} \approx-a_{4} w^{2}[\operatorname{cf}$. (B.12) $]$ in (B.10) leads to

$$
\tilde{J}\left(\mathbf{w}_{\text {stripeII }}, \tilde{\tau}_{4}\right) \approx-a_{2} w A_{3} \quad \text { with } A_{3}=\left(\begin{array}{ccc}
O & O & O \\
O & O & \hat{S} \\
O & \hat{S} & O
\end{array}\right), \quad \hat{S}=\left(\begin{array}{ll}
0 & 1 \\
1 & 0
\end{array}\right) .
$$

The eigenvalues of the matrix $\tilde{J}\left(\mathbf{w}_{\text {stripeII }}, \tilde{\tau}_{4}\right)$ are $\lambda_{1} \approx-a_{2} w, \lambda_{2} \approx a_{2} w$, and $\lambda_{3} \approx 0$ (repeated twice). Since the eigenvalues $\lambda_{1}$ and $\lambda_{2}$ have opposite signs, the associated bifurcating solution is always asymptotically unstable.

\section{B.4. Bifurcation point with $M=12$}

For $\mu=(12 ; k, \ell)$, we define complex variables

$$
\mathbf{z}=\left(z_{1}, \ldots, z_{6}\right)=\left(w_{1}+\mathrm{i} w_{2}, \ldots, w_{11}+\mathrm{i} w_{12}\right)
$$

and a system of bifurcation equation in complex variables as

$$
F_{i}\left(z_{1}, \ldots, z_{6}, \bar{z}_{1}, \ldots, \bar{z}_{6}, \tilde{\tau}\right)=0, \quad i=1, \ldots, 6 .
$$

The function $F_{1}\left(z_{1}, \ldots, z_{6}, \bar{z}_{1}, \ldots, \bar{z}_{6}, \tilde{\tau}\right)$ can be expanded into a power series as

$$
F_{1}\left(z_{1}, \ldots, z_{6}, \bar{z}_{1}, \ldots, \bar{z}_{6}, \tilde{\tau}\right)=\sum_{a=0} \sum_{b=0} \cdots \sum_{u=0} A_{a b c d e g h i j s t u}(\tilde{\tau}) z_{1}^{a} z_{2}^{b} z_{3}^{c} z_{4}^{d} z_{5}^{e} z_{6}^{g} \bar{z}_{1}^{h} \bar{z}_{2}^{i} \bar{z}_{3}^{j} \bar{z}_{4}^{s} \bar{z}_{5}^{t} \bar{z}_{6}^{u} .
$$

Since $\left(z_{1}, \ldots, z_{6}, \bar{z}_{1}, \ldots, \bar{z}_{6}, \tilde{\tau}\right)=(0, \ldots, 0)$ corresponds to the bifurcation point of multiplicity 12 , we have

$$
A_{000000000000}(0)=0, \quad A_{100000000000}(0)=A_{010000000000}(0)=\cdots=A_{000000000001}(0)=0 .
$$


By the equivariance conditions for $r$ and $s$, we have $A_{a b \ldots t u}(\tilde{\tau}) \in \mathbb{R}$ and

$$
\begin{aligned}
& F_{2}\left(z_{1}, z_{2}, z_{3}, z_{4}, z_{5}, z_{6}\right)=F_{1}\left(z_{2}, z_{3}, z_{1}, z_{6}, z_{4}, z_{5}\right), \\
& F_{3}\left(z_{1}, z_{2}, z_{3}, z_{4}, z_{5}, z_{6}\right)=F_{1}\left(z_{3}, z_{1}, z_{2}, z_{5}, z_{6}, z_{4}\right), \\
& F_{4}\left(z_{1}, z_{2}, z_{3}, z_{4}, z_{5}, z_{6}\right)=F_{1}\left(z_{4}, z_{5}, z_{6}, z_{1}, z_{2}, z_{3}\right), \\
& F_{5}\left(z_{1}, z_{2}, z_{3}, z_{4}, z_{5}, z_{6}\right)=F_{1}\left(z_{5}, z_{6}, z_{4}, z_{3}, z_{1}, z_{2}\right), \\
& F_{6}\left(z_{1}, z_{2}, z_{3}, z_{4}, z_{5}, z_{6}\right)=F_{1}\left(z_{6}, z_{4}, z_{5}, z_{2}, z_{3}, z_{1}\right),
\end{aligned}
$$

in which $\bar{z}_{1}, \ldots, \bar{z}_{6}$, and $\tilde{\tau}$ are suppressed. By the equivariance with respect to $p_{1}$ and $p_{2}$, we have the condition for the presence of the term $A_{a b \ldots t u}(\tilde{\tau})$ as

$$
\begin{aligned}
& \hat{k}(a-h)+\hat{\ell}(b-i)-(\hat{k}+\hat{\ell})(c-j)+\hat{k}(d-s)+\hat{\ell}(e-t)-(\hat{k}+\hat{\ell})(g-u) \equiv \hat{k} \bmod \hat{n}, \\
& \hat{\ell}(a-h)-(\hat{k}+\hat{\ell})(b-i)+\hat{k}(c-j)-(\hat{k}+\hat{\ell})(d-s)+\hat{k}(e-t)+\hat{\ell}(g-u) \equiv \hat{\ell} \bmod \hat{n},
\end{aligned}
$$

where $\hat{k}=\frac{k}{\operatorname{gcd}(k, \ell, n)}, \hat{\ell}=\frac{\ell}{\operatorname{gcd}(k, \ell, n)}$, and $\hat{n}=\frac{n}{\operatorname{gcd}(k, \ell, n)}$.

In the study of the stability of bifurcating solutions, it is sufficient to consider the power up to the order of two, i.e.

$$
F_{1} \approx \begin{cases}a_{1} \tilde{\tau} z_{1}+\left(a_{2} \bar{z}_{2} \bar{z}_{3}+a_{3} \bar{z}_{1} z_{3}+a_{4} z_{2}^{2}\right) & \text { for }(\hat{k}, \hat{\ell}, \hat{n})=(2,1,7), \\ a_{1} \tilde{\tau} z_{1}+a_{2} \bar{z}_{2} \bar{z}_{3} & \text { for }(\hat{k}, \hat{\ell}, \hat{n}) \neq(2,1,7)\end{cases}
$$

(see Appendix D for the proof). Candidates of bifurcating solutions are $(w \in \mathbb{R})$

$$
\begin{array}{ll}
\mathbf{w}_{\text {hexaVM }}=(w, 0, w, 0, w, 0, w, 0, w, 0, w, 0), & \mathbf{w}_{\text {racetrack }}=-\mathbf{w}_{\text {hexaVM }}, \\
\mathbf{w}_{\text {hexaT }}=(w, 0, w, 0, w, 0,0,0,0,0,0,0), & \mathbf{w}_{\text {racetrack }}=-\mathbf{w}_{\text {hexaT }}, \\
\mathbf{w}_{\text {stripeI }}=(w, 0,0,0,0,0,0,0,0,0,0,0), & \mathbf{w}_{\text {upside-downI }}=(w, 0,0,0,0,0, w, 0,0,0,0,0), \\
\mathbf{w}_{\text {stripeII }}=(0, w, 0,0,0,0,0,0,0,0,0,0), & \mathbf{w}_{\text {upside-downII }}=(0, w, 0,0,0,0,0, w, 0,0,0,0) .
\end{array}
$$

B.4.1. Case 1: $(\hat{k}, \hat{\ell}, \hat{n})=(2,1,7)$

For $(\hat{n}, \hat{k}, \hat{\ell})=(7,2,1)$, from (B.16) and (B.19), we have

$$
\begin{array}{ll}
F_{1} \approx a_{1} \tilde{\tau} z_{1}+\left(a_{2} \bar{z}_{2} \bar{z}_{3}+a_{3} z_{3} \bar{z}_{1}+a_{4} z_{2}^{2}\right), & F_{4} \approx a_{1} \tilde{\tau} z_{4}+\left(a_{2} \bar{z}_{5} \bar{z}_{6}+a_{3} z_{6} \bar{z}_{4}+a_{4} z_{5}^{2}\right), \\
F_{2} \approx a_{1} \tilde{\tau} z_{2}+\left(a_{2} \bar{z}_{3} \bar{z}_{1}+a_{3} z_{1} \bar{z}_{2}+a_{4} z_{3}^{2}\right), & F_{5} \approx a_{1} \tilde{\tau} z_{5}+\left(a_{2} \bar{z}_{6} \bar{z}_{4}+a_{3} z_{4} \bar{z}_{5}+a_{4} z_{6}^{2}\right), \\
F_{3} \approx a_{1} \tilde{\tau} z_{3}+\left(a_{2} \bar{z}_{1} \bar{z}_{2}+a_{3} z_{2} \bar{z}_{3}+a_{4} z_{1}^{2}\right), & F_{6} \approx a_{1} \tilde{\tau} z_{6}+\left(a_{2} \bar{z}_{4} \bar{z}_{5}+a_{3} z_{5} \bar{z}_{6}+a_{4} z_{4}^{2}\right) .
\end{array}
$$

The Jacobian matrix takes the form: $\tilde{J}(\mathbf{w}, \tilde{\tau}) \approx \operatorname{diag}\left(B_{1}, B_{2}\right)$, where

$$
B_{1}=\left(\begin{array}{cccccc}
a_{1} \tilde{\tau}+a_{3} w_{5} & a_{3} w_{6} & a_{2} w_{5}+2 a_{4} w_{3} & -a_{2} w_{6}-2 a_{4} w_{4} & a_{2} w_{3}+a_{3} w_{1} & -a_{2} w_{4}+a_{3} w_{2} \\
a_{3} w_{6} & a_{1} \tilde{\tau}-a_{3} w_{5} & -a_{2} w_{6}+2 a_{4} w_{4} & -a_{2} w_{5}+2 a_{4} w_{3} & -a_{2} w_{4}-a_{3} w_{2} & -a_{2} w_{3}+a_{3} w_{1} \\
a_{2} w_{5}+a_{3} w_{3} & -a_{2} w_{6}+a_{3} w_{4} & a_{1} \tilde{\tau}+a_{3} w_{1} & a_{3} w_{2} & a_{2} w_{1}+2 a_{4} w_{5} & -a_{2} w_{2}-2 a_{4} w_{6} \\
-a_{2} w_{6}-a_{3} w_{4} & -a_{2} w_{5}+a_{3} w_{3} & a_{3} w_{2} & a_{1} \tilde{\tau}-a_{3} w_{1} & -a_{2} w_{2}+2 a_{4} w_{6} & -a_{2} w_{1}+2 a_{4} w_{5} \\
a_{2} w_{3}+2 a_{4} w_{1} & -a_{2} w_{4}-2 a_{4} w_{2} & a_{2} w_{1}+a_{3} w_{5} & -a_{2} w_{2}+a_{3} w_{6} & a_{1} \tilde{\tau}+a_{3} w_{3} & a_{3} w_{4} \\
-a_{2} w_{4}+2 a_{4} w_{2} & -a_{2} w_{3}+2 a_{4} w_{1} & -a_{2} w_{2}-a_{3} w_{6} & -a_{2} w_{1}+a_{3} w_{5} & a_{3} w_{4} & a_{1} \tilde{\tau}-a_{3} w_{3}
\end{array}\right),
$$




$$
B_{2}=\left(\begin{array}{cccccc}
a_{1} \tilde{\tau}+a_{3} w_{11} & a_{3} w_{12} & a_{2} w_{11}+2 a_{4} w_{9} & -a_{2} w_{12}-2 a_{4} w_{10} & a_{2} w_{9}+a_{3} w_{7} & -a_{2} w_{10}+a_{3} w_{8} \\
a_{3} w_{12} & a_{1} \tilde{\tau}-a_{3} w_{11} & -a_{2} w_{12}+2 a_{4} w_{10} & -a_{2} w_{11}+2 a_{4} w_{9} & -a_{2} w_{10}-a_{3} w_{8} & -a_{2} w_{9}+a_{3} w_{7} \\
a_{2} w_{11}+a_{3} w_{9} & -a_{2} w_{12}+a_{3} w_{10} & a_{1} \tilde{\tau}+a_{3} w_{7} & a_{3} w_{8} & a_{2} w_{7}+2 a_{4} w_{11} & -a_{2} w_{8}-2 a_{4} w_{12} \\
-a_{2} w_{12}-a_{3} w_{10} & -a_{2} w_{11}+a_{3} w_{9} & a_{3} w_{8} & a_{1} \tilde{\tau}-a_{3} w_{7} & -a_{2} w_{8}+2 a_{4} w_{12} & -a_{2} w_{7}+2 a_{4} w_{11} \\
a_{2} w_{9}+2 a_{4} w_{7} & -a_{2} w_{10}-2 a_{4} w_{8} & a_{2} w_{7}+a_{3} w_{11} & -a_{2} w_{8}+a_{3} w_{12} & a_{1} \tilde{\tau}+a_{3} w_{9} & a_{3} w_{10} \\
-a_{2} w_{10}+2 a_{4} w_{8} & -a_{2} w_{9}+2 a_{4} w_{7} & -a_{2} w_{8}-a_{3} w_{12} & -a_{2} w_{7}+a_{3} w_{11} & a_{3} w_{10} & a_{1} \tilde{\tau}-a_{3} w_{9}
\end{array}\right) .
$$

For the present case with $(\hat{k}, \hat{\ell}, \hat{n})=(2,1,7)$, the stripe and upside-down patterns do not exist (see Examples C.1 and C.2 in Appendix C); accordingly, we focus on hexagonal patterns and associated racetrack patterns hereinafter.

For Type VM hexagonal pattern and associated racetrack pattern, the use of $\mathbf{w}_{\text {hexaVM }}=$ $(w, 0, \ldots, w, 0)$, i.e. $\mathbf{z}_{\text {hexaVM }}=(w, \ldots, w)$ in (B.20) gives

$$
F_{1}\left(\mathbf{z}_{\mathrm{hexaVM}}, \tilde{\tau}\right)=\cdots=F_{6}\left(\mathbf{z}_{\mathrm{hexaVM}}, \tilde{\tau}\right) \approx a_{1} \tilde{\tau} w+\left(a_{2}+a_{3}+a_{4}\right) w^{2}
$$

Thus there is a bifurcating solution: $a_{1} \tilde{\tau}_{1} \approx-\left(a_{2}+a_{3}+a_{4}\right) w$, and (B.21) and (B.22) become

$$
\begin{aligned}
& B_{1}\left(\mathbf{w}_{\text {hexaVM }}, \tilde{\tau}_{1}\right) \\
& =B_{2}\left(\mathbf{w}_{\text {hexaVM }}, \tilde{\tau}_{1}\right) \\
& \approx w\left(\begin{array}{cccccc}
-\left(a_{2}+a_{4}\right) & 0 & a_{2}+2 a_{4} & 0 & a_{2}+a_{3} & 0 \\
0 & -\left(a_{2}+2 a_{3}+a_{4}\right) & 0 & -a_{2}+2 a_{4} & 0 & -a_{2}+a_{3} \\
a_{2}+a_{3} & 0 & -\left(a_{2}+a_{4}\right) & 0 & a_{2}+2 a_{4} & 0 \\
0 & -a_{2}+a_{3} & 0 & -\left(a_{2}+2 a_{3}+a_{4}\right) & 0 & -a_{2}+2 a_{4} \\
a_{2}+2 a_{4} & 0 & a_{2}+a_{3} & 0 & -\left(a_{2}+a_{4}\right) & 0 \\
0 & -a_{2}+2 a_{4} & 0 & -a_{2}+a_{3} & 0 & -\left(a_{2}+2 a_{3}+a_{4}\right)
\end{array}\right) .
\end{aligned}
$$

The eigenvalues are repeated twice and are given by

$$
\begin{aligned}
& \lambda_{1} \approx\left(a_{2}+a_{3}+a_{4}\right) w, \quad \lambda_{2}, \lambda_{3} \approx\left[-\left(2 a_{2}+\frac{a_{3}}{2}+2 a_{4}\right) \pm \mathrm{i} \frac{\sqrt{3}}{2}\left(2 a_{4}-a_{3}\right)\right] w, \\
& \lambda_{4} \approx-\left(3 a_{2}+a_{3}-a_{4}\right) w, \quad \lambda_{5}, \lambda_{6} \approx\left[-\left(\frac{5}{2} a_{3}+2 a_{4}\right) \pm \mathrm{i} \frac{\sqrt{3}}{2}\left(2 a_{4}-a_{3}\right)\right] w .
\end{aligned}
$$

Since the real parts of at least one of the eigenvalues $\lambda_{1}, \ldots, \lambda_{6}$ are positive by Lemma[1]below, the bifurcating hexagonal solution is unstable. Similarly, the associated racetrack pattern is unstable.

Lemma 1. The following four inequalities are not satisfied simultaneously:

$$
\begin{gathered}
\frac{\operatorname{Re}\left(\lambda_{1}\right)}{w}=a_{2}+a_{3}+a_{4}<0, \\
-2 \frac{\operatorname{Re}\left(\lambda_{2}\right)}{w}=4 a_{2}+a_{3}+4 a_{4}>0 \\
-\frac{\operatorname{Re}\left(\lambda_{4}\right)}{w}=3 a_{2}+a_{3}-a_{4}>0, \\
-2 \frac{\operatorname{Re}\left(\lambda_{5}\right)}{w}=5 a_{3}+4 a_{4}>0 .
\end{gathered}
$$

Proof. We first consider the case of $w>0$, i.e. 
Then we have

$$
\left\{\begin{array}{l}
\text { From }(\text { B.26) with }(\underline{B .25}): \\
\quad 3 a_{3}<4\left(a_{2}+a_{3}+a_{4}\right)<0 \Rightarrow a_{3}<0, \\
\text { From (B.27) with }(\underline{B .25}): \\
\quad 2 a_{3}+4 a_{4}<3\left(a_{2}+a_{3}+a_{4}\right)<0 .
\end{array}\right.
$$

Then from (B.28) and B.29), we have $2 a_{3}+4 a_{4}<$ $5 a_{3}+4 a_{4} \Rightarrow a_{3}>0$. Thus we have a contradiction with respect to the sign of $a_{3}$, thereby showing that there is at least one positive eigenvalue. Equation (B.24) for the case of $w<0$ can be shown similarly.

Type $\mathrm{T}$ hexagonal pattern and associated racetrack pattern exist for the present case of $(\hat{k}, \hat{\ell}, \hat{n})=$ $(2,1,7)$, which satisfies the condition (11). The use of $\mathbf{w}_{\text {hexaT }}=(w, 0, w, 0, w, 0,0,0,0,0,0,0)$, i.e. $\mathbf{z}_{\text {hexaT }}=(w, w, w, 0,0,0)$ in (B.20) gives

$$
\left\{\begin{aligned}
F_{1}\left(\mathbf{z}_{\text {hexaT }}, \tilde{\tau}\right) & =F_{2}\left(\mathbf{z}_{\text {hexaT }}, \tilde{\tau}\right)=F_{3}\left(\mathbf{z}_{\text {hexaT }}, \tilde{\tau}\right) \\
& \approx a_{1} \tilde{\tau} w+\left(a_{2}+a_{3}+a_{4}\right) w^{2}, \\
F_{4}\left(\mathbf{z}_{\text {hexaT }}, \tilde{\tau}\right) & =F_{5}\left(\mathbf{z}_{\text {hexaT }}, \tilde{\tau}\right)=F_{6}\left(\mathbf{z}_{\text {hexaT }}, \tilde{\tau}\right)=0
\end{aligned}\right.
$$

Ikeda \& Murota, 2014, p. 302]. We have a bifurcating solution $a_{1} \tilde{\tau}_{1} \approx-\left(a_{2}+a_{3}+a_{4}\right) w$, and B.21) and (B.22) become

$$
\begin{aligned}
& B_{1}\left(\mathbf{w}_{\text {hexaT }}, \tilde{\tau}_{1}\right)=B_{1}\left(\mathbf{w}_{\text {hexaVM }}, \tilde{\tau}_{1}\right), \\
& B_{2}\left(\mathbf{w}_{\text {hexaT }}, \tilde{\tau}_{1}\right) \approx-\left(a_{2}+a_{3}+a_{4}\right) w I .
\end{aligned}
$$

Since the eigenvalues of $B_{1}\left(\mathbf{w}_{\text {hexaT }}, \tilde{\tau}_{1}\right)$ are given by those of $B_{1}\left(\mathbf{w}_{\text {hexaVM }}, \tilde{\tau}_{1}\right)$ in (B.23) for Type VM, the bifurcating hexagonal solution is unstable. Similarly, the associated racetrack pattern is unstable.

\section{B.4.2. Case 2: $(\hat{k}, \hat{\ell}, \hat{n}) \neq(2,1,7)$}

For $(\hat{k}, \hat{\ell}, \hat{n}) \neq(2,1,7)$, from (B.16) and (B.19), we have

$$
\begin{aligned}
& F_{1}(\mathbf{z}, \tilde{\tau}) \approx a_{1} \tilde{\tau} z_{1}+a_{2} \bar{z}_{2} \bar{z}_{3}, \\
& F_{2}(\mathbf{z}, \tilde{\tau}) \approx a_{1} \tilde{\tau} z_{2}+a_{2} \bar{z}_{3} \bar{z}_{1}, \\
& F_{3}(\mathbf{z}, \tilde{\tau}) \approx a_{1} \tilde{\tau} z_{3}+a_{2} \bar{z}_{1} \bar{z}_{2}, \\
& F_{4}(\mathbf{z}, \tilde{\tau}) \approx a_{1} \tilde{\tau} z_{4}+a_{2} \bar{z}_{5} \bar{z}_{6}, \\
& F_{5}(\mathbf{z}, \tilde{\tau}) \approx a_{1} \tilde{\tau} z_{5}+a_{2} \bar{z}_{6} \bar{z}_{4}, \\
& F_{6}(\mathbf{z}, \tilde{\tau}) \approx a_{1} \tilde{\tau} z_{6}+a_{2} \bar{z}_{4} \bar{z}_{5},
\end{aligned}
$$

and $\tilde{J}(\mathbf{w}, \tilde{\tau}) \approx\left(\begin{array}{cc}C_{1} & O \\ O & C_{2}\end{array}\right)$ with

$$
\begin{gathered}
C_{1}(\mathbf{w}, \tilde{\tau}) \equiv\left(\begin{array}{cccccc}
a_{1} \tilde{\tau} & 0 & a_{2} w_{5} & -a_{2} w_{6} & a_{2} w_{3} & -a_{2} w_{4} \\
0 & a_{1} \tilde{\tau} & -a_{2} w_{6} & -a_{2} w_{5} & -a_{2} w_{4} & -a_{2} w_{3} \\
a_{2} w_{5} & -a_{2} w_{6} & a_{1} \tilde{\tau} & 0 & a_{2} w_{1} & -a_{2} w_{2} \\
-a_{2} w_{6} & -a_{2} w_{5} & 0 & a_{1} \tilde{\tau} & -a_{2} w_{2} & -a_{2} w_{1} \\
a_{2} w_{3} & -a_{2} w_{4} & a_{2} w_{1} & -a_{2} w_{2} & a_{1} \tilde{\tau} & 0 \\
-a_{2} w_{4} & -a_{2} w_{3} & -a_{2} w_{2} & -a_{2} w_{1} & 0 & a_{1} \tilde{\tau}
\end{array}\right) \\
C_{2}(\mathbf{w}, \tilde{\tau}) \equiv\left(\begin{array}{ccccccc}
a_{1} \tilde{\tau} & 0 & a_{2} w_{11} & -a_{2} w_{12} & a_{2} w_{9} & -a_{2} w_{10} \\
0 & a_{1} \tilde{\tau} & -a_{2} w_{12} & -a_{2} w_{11} & -a_{2} w_{10} & -a_{2} w_{9} \\
a_{2} w_{11} & -a_{2} w_{12} & a_{1} \tilde{\tau} & 0 & a_{2} w_{7} & -a_{2} w_{8} \\
-a_{2} w_{12} & -a_{2} w_{11} & 0 & a_{1} \tilde{\tau} & -a_{2} w_{8} & -a_{2} w_{7} \\
a_{2} w_{9} & -a_{2} w_{10} & a_{2} w_{7} & -a_{2} w_{8} & a_{1} \tilde{\tau} & 0 \\
-a_{2} w_{10} & -a_{2} w_{9} & -a_{2} w_{8} & -a_{2} w_{7} & 0 & a_{1} \tilde{\tau}
\end{array}\right) .
\end{gathered}
$$

For Type VM hexagonal pattern, the use of $\mathbf{w}_{\text {hexaVM }}=(w, 0, \ldots, w, 0)$, i.e. $\mathbf{z}_{\text {hexaVM }}=(w, \ldots, w)$ in (B.30) gives

$$
F_{1}\left(\mathbf{z}_{\text {hexaVM }}, \tilde{\tau}\right)=\cdots=F_{6}\left(\mathbf{z}_{\text {hexaVM }}, \tilde{\tau}\right) \approx a_{1} \tilde{\tau} w+a_{2} w^{2} .
$$

Thus the bifurcation equation has a bifurcating solution: $a_{1} \tilde{\tau}_{2} \approx-a_{2} w$, and (B.31) becomes

$$
C_{1}\left(\mathbf{w}_{\text {hexaVM }}, \tilde{\tau}_{2}\right)=C_{2}\left(\mathbf{w}_{\text {hexaVM }}, \tilde{\tau}_{2}\right)=a_{2} w A_{1} .
$$


The bifurcating solutions are unstable because these matrices are equal to the Jacobian matrix (B.11) that have eigenvalues with opposite signs.

Type T hexagonal pattern exists when the condition (11) is satisfied. The use of $\mathbf{w}_{\text {hexaT }}=(w, 0$, $w, 0, w, 0,0,0,0,0,0,0)$, i.e. $\mathbf{z}_{\text {hexaT }}=(w, w, w, 0$, $0,0)$ in $(\underline{B} .20)$ gives

$$
\left\{\begin{aligned}
F_{1}\left(\mathbf{z}_{\text {hexaT }}, \tilde{\tau}\right) & =F_{2}\left(\mathbf{z}_{\text {hexaT }}, \tilde{\tau}\right)=F_{3}\left(\mathbf{z}_{\text {hexaT }}, \tilde{\tau}\right) \\
& \approx a_{1} \tilde{\tau} w+a_{2} w^{2}+\text { h.o.t., } \\
F_{4}\left(\mathbf{z}_{\text {hexaT }}, \tilde{\tau}\right) & =F_{5}\left(\mathbf{z}_{\text {hexaT }}, \tilde{\tau}\right)=F_{z}\left(\mathbf{w}_{\text {hexaT }}, \tilde{\tau}\right)=0
\end{aligned}\right.
$$

Ikeda \& Murota, 2014, p. 302]. Thus the bifurcation equation has a bifurcating solution: $a_{1} \tilde{\tau}_{2} \approx-a_{2} w$, and (B.31) becomes

$$
\begin{aligned}
& C_{1}\left(\mathbf{w}_{\text {hexaT }}, \tilde{\tau}_{2}\right) \approx a_{2} w A_{1}, \\
& C_{2}\left(\mathbf{w}_{\text {hexaT }}, \tilde{\tau}_{2}\right) \approx-a_{2} w I .
\end{aligned}
$$

Since the matrix $C_{1}\left(\mathbf{w}_{\text {hexaT }}, \tilde{\tau}_{2}\right)$ is equal to the Jacobian matrix (B.11) for the sextuple bifurcation point, the bifurcating solutions for the present case are also unstable.

Type I stripe pattern exists when the condition (12) is satisfied. For $\mathbf{w}_{\text {stripeI }}=(w, 0, \ldots, 0)$, i.e. $\mathbf{z}_{\text {stripeI }}=(w, 0,0,0,0,0)$, we have (see Appendix C.1)

$$
\left\{\begin{array}{l}
F_{1}\left(\mathbf{z}_{\text {stripeI }}, \tilde{\tau}\right) \approx a_{1} \tilde{\tau} w+a_{5} w^{3}, \\
F_{i}\left(\mathbf{z}_{\text {stripeI }}, \tilde{\tau}\right)=0, \quad i=2, \ldots, 6
\end{array}\right.
$$

$\left(a_{5}=A_{200000100000}(0)\right)$. Thus the bifurcation equation has a bifurcating solution: $a_{1} \tilde{\tau}_{3} \approx-a_{5} w^{2}$, and (B.31) becomes

$$
C_{1}\left(\mathbf{w}_{\text {stripeI }}, \tilde{\tau}_{3}\right) \approx a_{2} w A_{2}, \quad C_{2}\left(\mathbf{w}_{\text {stripeI }}, \tilde{\tau}_{3}\right) \approx O
$$

with $A_{2}$ in $(\underline{B .13})$ and $a_{5}(0) w^{2}$ has been omitted as a higher order term than $a_{2} w$. The eigenvalues are $\lambda_{1} \approx-a_{2} w, \lambda_{2} \approx a_{2} w$, and so on. Since $\lambda_{1}$ and $\lambda_{2}$ have opposite signs, the bifurcating solution is asymptotically unstable for any $a_{2} w$.

Type II stripe pattern exists when $\hat{n}$ is even and the condition (12) is satisfied. For $\mathbf{w}_{\text {stripeII }}=$ $(0, w, 0, \ldots, 0)$, i.e. $\mathbf{z}_{\text {stripeII }}=(\mathrm{i} w, 0,0,0,0,0)$, we have (see Appendix C.1)

$$
\left\{\begin{array}{l}
F_{1}\left(\mathbf{z}_{\text {stripeII }}, \tilde{\tau}\right) \approx \mathrm{i}\left(a_{1} \tilde{\tau} w+a_{5} w^{3}\right) \\
F_{i}\left(\mathbf{z}_{\text {stripeII }}, \tilde{\tau}\right)=0, \quad i=2, \ldots, 6
\end{array}\right.
$$

Thus the bifurcation equation has a bifurcating solution: $a_{1} \tilde{\tau}_{3} \approx-a_{5} w^{2}$. Then (B.31) gives

$C_{1}\left(\mathbf{w}_{\text {stripeII }}, \tilde{\tau}_{3}\right) \approx-a_{2} w A_{3}, \quad C_{2}\left(\mathbf{w}_{\text {stripeII }}, \tilde{\tau}_{3}\right) \approx O$

with $A_{3}$ in (B.14). The eigenvalues are $\lambda_{1} \approx-a_{2} w$, $\lambda_{2} \approx a_{2} w$, and so on. Since $\lambda_{1}$ and $\lambda_{2}$ have opposite signs, the bifurcating solution is asymptotically unstable for any $a_{2} w$.

Type I upside-down pattern exists when the condition (13) is satisfied. For $\mathbf{w}_{\text {upside-downI }}=(w, 0$, $0,0,0,0, w, 0,0,0,0,0)$, i.e. $\mathbf{z}_{\text {upside-downI }}=(w, 0,0$, $w, 0,0$ ), we have (see Appendix C.2)

$$
\left\{\begin{aligned}
F_{1}\left(\mathbf{z}_{\text {upside-downI }}, \tilde{\tau}\right) & =F_{4}\left(\mathbf{z}_{\text {upside-downI }}, \tilde{\tau}\right) \\
& \approx a_{1} \tilde{\tau} w+a_{6} w^{3} \\
F_{i}\left(\mathbf{z}_{\text {upside-downI }}, \tilde{\tau}\right) & =0, \quad i=2,3,5,6 .
\end{aligned}\right.
$$

Thus the bifurcation equation has a bifurcating solution: $a_{1} \tilde{\tau}_{4} \approx-a_{6} w^{2}$. Then (B.31) gives

$$
\begin{aligned}
C_{1}\left(\mathbf{w}_{\text {upside-downI }}, \tilde{\tau}_{4}\right) & =C_{2}\left(\mathbf{w}_{\text {upside-downI }}, \tilde{\tau}_{4}\right) \\
& \approx a_{2} w A_{2}
\end{aligned}
$$

with $A_{2}$ in B.13). The eigenvalues are $\lambda_{1} \approx-a_{2} w$, $\lambda_{2} \approx a_{2} w$, and so on. Since $\lambda_{1}$ and $\lambda_{2}$ have opposite signs, the bifurcating solution is asymptotically unstable for any $a_{2} w$.

Type II upside-down pattern exists when $\hat{n}$ is even and the condition (13) is satisfied. For $\mathbf{w}_{\text {upside-downII }}=(0, w, 0,0,0,0,0, w, 0,0,0,0)$, i.e. $\mathbf{z}_{\text {upside-downII }}=(\mathrm{i} w, 0,0, \mathrm{i} w, 0,0)$, we have (see Appendix C.2)

$$
\left\{\begin{aligned}
\frac{F_{1}\left(\mathbf{z}_{\text {upside-downII }}, \tilde{\tau}\right)}{i} & =\frac{F_{4}\left(\mathbf{z}_{\text {upside-downII }}, \tilde{\tau}\right)}{i \in \mathbb{R}}, \\
F_{1}\left(\mathbf{z}_{\text {upside-downII }}, \tilde{\tau}\right) & =F_{4}\left(\mathbf{z}_{\text {upside-downII }}, \tilde{\tau}\right) \\
& \approx \mathrm{i}\left(a_{1} \tilde{\tau} w+a_{6} w^{3}\right) \\
F_{i}\left(\mathbf{z}_{\text {upside-downII }}, \tilde{\tau}\right) & =0, \quad i=2,3,5,6 .
\end{aligned}\right.
$$

Thus the bifurcation equation has a bifurcating solution: $a_{1} \tilde{\tau}_{4} \approx-a_{6} w^{2}$. Then (B.31) gives

$$
\begin{aligned}
C_{1}\left(\mathbf{w}_{\text {upside-downII }}, \tilde{\tau}_{4}\right) & =C_{2}\left(\mathbf{w}_{\text {upside-downII }}, \tilde{\tau}_{4}\right) \\
& \approx a_{2} w A_{3}
\end{aligned}
$$

with $A_{3}$ in (B.14). The eigenvalues are $\lambda_{1} \approx-a_{2} w$, $\lambda_{2} \approx a_{2} w$, and so on. Since $\lambda_{1}$ and $\lambda_{2}$ have opposite signs, the bifurcating solution is asymptotically unstable for any $a_{2} w$. 


\section{Appendix C}

\section{Existence of Stripe and Upside-Down}

Patterns for $\mu=(12 ; k, \ell)$

\section{C.1. Stripe patterns}

The use of $(a, b, c, d, e, g, h, i, j, s, t, u)=(a, 0,0,0$, $0,0, h, 0,0,0,0,0)$ in (B.17) and (B.18) leads to $a=1+h+p \hat{n}(p \in \mathbb{Z})$. Then $F_{1}$ in $(\mathbb{B} .15)$ is rewritten as

$$
\begin{aligned}
& F_{1}\left(z_{1}, 0,0,0,0,0, \tilde{\tau}\right) \\
& =\sum_{q=0}^{\infty} A_{1+q, q}(\tilde{\tau})\left|z_{1}\right|^{2 q} z_{1} \\
& \quad+\sum_{p=1}^{\infty} \sum_{q=0}^{\infty}\left[A_{q+1+p \hat{n}, q}(\tilde{\tau})\left|z_{1}\right|^{2 q} z_{1}^{p \hat{n}+1}\right. \\
& \left.\quad+A_{q, q-1+p \hat{n}}(\tilde{\tau})\left|z_{1}\right|^{2 q} \bar{z}_{1}^{p \hat{n}-1}\right] \\
& \approx A_{10}^{\prime}(0) \tilde{\tau} z_{1}+A_{21}(0)\left|z_{1}\right|^{2} z_{1}
\end{aligned}
$$

$\left(A_{a h}=A_{a 00000 h 00000}(0)\right)$. We hereafter consider two kinds of stripe patterns $\left(z_{1}, \ldots, z_{6}\right)=(w, 0,0$, $0,0,0)$ and $\left(z_{1}, \ldots, z_{6}\right)=(\mathrm{i} w, 0,0,0,0,0)$.

For Type I stripe pattern with $\left(z_{1}, \ldots, z_{6}\right)=$ $(w, 0,0,0,0,0)$, C.1 becomes

$$
\begin{aligned}
& F_{1}(w, 0,0,0,0,0, \tilde{\tau}) \in \mathbb{R}, \\
& F_{1}(w, 0,0,0,0,0, \tilde{\tau}) \approx a_{1} \tilde{\tau} w+a_{5} w^{3}
\end{aligned}
$$

$\left(a_{1}=A_{10}^{\prime}(0)\right.$ and $\left.a_{5}=A_{21}(0)\right)$ and (B.16) becomes

$$
\begin{aligned}
& F_{2}(w, 0,0,0,0,0)=F_{1}(0,0, w, 0,0,0), \\
& F_{3}(w, 0,0,0,0,0)=F_{1}(0, w, 0,0,0,0), \\
& F_{4}(w, 0,0,0,0,0)=F_{1}(0,0,0, w, 0,0), \\
& F_{5}(w, 0,0,0,0,0)=F_{1}(0,0,0,0, w, 0), \\
& F_{6}(w, 0,0,0,0,0)=F_{1}(0,0,0,0,0, w) .
\end{aligned}
$$

With the use of $T=\left\{(a, b, \ldots, t, u) \in \mathbb{N}_{+}^{12} \mid\right.$ (B.17), (B.18) $\}, F_{2}=\cdots=F_{6}=0$ in C.2 are satisfied if

$$
\begin{aligned}
& (0, b, 0,0,0,0,0, i, 0,0,0,0) \notin T, \\
& (0,0, c, 0,0,0,0,0, j, 0,0,0) \notin T, \\
& (0,0,0, d, 0,0,0,0,0, s, 0,0) \notin T, \\
& (0,0,0,0, e, 0,0,0,0,0, t, 0) \notin T, \\
& (0,0,0,0,0, g, 0,0,0,0,0, u) \notin T .
\end{aligned}
$$

We first consider the case of $(a, b, \ldots, t, u)=$ $(0, b, 0,0,0,0,0, i, 0,0,0,0)$. For this case, B.17) and (B.18) become

$$
\left\{\begin{array}{l}
\hat{\ell} \alpha-p \hat{n}=\hat{k} \\
-(\hat{k}+\hat{\ell}) \alpha-q \hat{n}=\hat{\ell}
\end{array} \quad(\alpha, p, q \in \mathbb{Z}),\right.
$$

where $\alpha=b-i$. This relation can be expressed in a matrix form as

$$
\begin{aligned}
A \mathbf{x}=\mathbf{b} \quad \text { with } A & =\left[\begin{array}{ccc}
\hat{\ell} & -\hat{n} & 0 \\
-(\hat{k}+\hat{\ell}) & 0 & -\hat{n}
\end{array}\right], \\
\mathbf{x} & =\left[\begin{array}{l}
\alpha \\
p \\
q
\end{array}\right], \quad \mathbf{b}=\left[\begin{array}{l}
\hat{k} \\
\hat{\ell}
\end{array}\right] .
\end{aligned}
$$

Remark C.1. Let $A$ be an $m \times n$ integer matrix and b an $m$-dimensional integer vector. The system of equations $A \mathrm{x}=\mathbf{b}$ admits an integer solution $\mathbf{x}$ if and only if two matrices $A$ and $[A \mid \mathbf{b}]$ share the same determinantal divisors, i.e.

$$
\operatorname{rank} A=\operatorname{rank}[A \mid \mathbf{b}], \quad d_{k}(A)=d_{k}([A \mid \mathbf{b}])
$$

for all $k$. Here $d_{k}(A)$ is the $k$ th determinantal divisor, which is the greatest common divisor of all $k \times k$ minors (subdeterminants) of the integer matrix $A$ Ikeda \& Murota, 2014, p. 257].

The existence of an integer solution $\mathrm{x}$ of (C.4) is investigated by showing the two conditions (C.5) in Remark C.1. The first condition $\operatorname{rank} A=$ $\operatorname{rank}[A \mid \mathbf{b}]$ is satisfied because

$$
\begin{aligned}
\operatorname{rank} A & =\operatorname{rank}\left[\begin{array}{ccc}
\hat{\ell} & -\hat{n} & 0 \\
-\hat{k} & 0 & -\hat{n}
\end{array}\right]=2, \\
\operatorname{rank}[A \mid \mathbf{b}] & =\operatorname{rank}\left[\begin{array}{cccc}
\hat{\ell} & -\hat{n} & 0 & \hat{k} \\
-\hat{k} & 0 & -\hat{n} & \hat{k}+\hat{\ell}
\end{array}\right]=2 .
\end{aligned}
$$

For the second condition, we have

$$
\begin{aligned}
d_{1}(A) & =\operatorname{gcd}(\hat{k}, \hat{\ell}, \hat{n})=1, \\
d_{1}([A \mid \mathbf{b}]) & =\operatorname{gcd}(\hat{k}, \hat{\ell}, \hat{n})=1, \\
d_{2}(A) & =\operatorname{gcd}\left(\hat{k} \hat{n}, \hat{\ell} \hat{n}, \hat{n}^{2}\right)=\operatorname{gcd}(\hat{k}, \hat{\ell}, \hat{n}) \hat{n}=\hat{n}, \\
d_{2}([A \mid \mathbf{b}]) & =\operatorname{gcd}\left(\hat{k} \hat{n}, \hat{\ell} \hat{n}, \hat{n}^{2}, \hat{k}^{2}+\hat{k} \hat{\ell}+\hat{\ell}^{2}\right) \\
& =\operatorname{gcd}\left(\hat{n}, \hat{k}^{2}+\hat{k} \hat{\ell}+\hat{\ell}^{2}\right) .
\end{aligned}
$$


Thus $d_{1}(A)=d_{1}([A \mid \mathbf{b}])$ is always satisfied, whereas $d_{2}(A)=d_{2}([A \mid \mathbf{b}])$ is satisfied if $\hat{k}^{2}+\hat{k} \hat{\ell}+\hat{\ell}^{2}$ is divisible by $\hat{n}$. Then (C.4) has an integer solution $\mathbf{x}$ and, in turn, we have $(0, b, 0,0,0,0,0, i, 0$, $0,0,0) \in T$, thereby leading to

$$
F_{3}(w, 0,0,0,0,0, \tilde{\tau})=F_{1}(0, w, 0,0,0,0, \tilde{\tau}) \neq 0 .
$$

On the contrary, $(0, b, 0,0,0,0,0, i, 0,0,0,0) \notin T$ and, in turn, $F_{3}=0$ is satisfied if the following condition holds

$$
\hat{k}^{2}+\hat{k} \hat{\ell}+\hat{\ell}^{2} \text { is not divisible by } \hat{n} \text {. }
$$

This is the first of the five conditions for (C.3). The remaining four conditions, respectively, give

$$
\begin{aligned}
\hat{k}^{2}+\hat{k} \hat{\ell}+\hat{\ell}^{2}, \hat{k}^{2}+2 \hat{k} \hat{\ell}, \hat{k}^{2}-\hat{\ell}^{2}, \\
\hat{\ell}^{2}+2 \hat{k} \hat{\ell} \text { are not divisible by } \hat{n} .
\end{aligned}
$$

This suffices to prove Proposition 3 .

For Type II stripe pattern with $\left(z_{1}, \ldots, z_{6}\right)=$ (iw, 0, 0, 0,0,0), C.1 becomes

$$
\begin{aligned}
& F_{1}(\mathrm{i} w, 0,0,0,0,0, \tilde{\tau}) \\
& =\mathrm{i} \sum_{q=0}^{\infty} A_{1+q, q}(\tilde{\tau}) w^{2 q+1} \\
& \quad+\sum_{p=1}^{\infty} \sum_{q=0}^{\infty}\left[\mathrm{i} A_{q+1+p \hat{n}, q}(\tilde{\tau}) \mathrm{i}^{p \hat{n}} w^{2 q+1+p \hat{n}}\right. \\
& \left.\quad-\mathrm{i} A_{q, q-1+p \hat{n}}(\tilde{\tau})(-\mathrm{i})^{p \hat{n}} w^{2 q-1+p \hat{n}}\right] \\
& \approx \mathrm{i}\left(a_{1} \tilde{\tau} w+a_{5} w^{3}\right) .
\end{aligned}
$$

Thus $F_{1}(w, 0,0,0,0,0, \tilde{\tau})=0$ can accommodate a solution curve if $\hat{n}$ is even ( $\mathrm{i}^{p \hat{n}}$ and $(-\mathrm{i})^{p \hat{n}}$ are real) and cannot accommodate it if $\hat{n}$ is odd. Then a discussion similar to that for stripe pattern I holds.

Example C.1. For $(\hat{k}, \hat{\ell})=(2,1)$, we have $\hat{k}^{2}+$ $\hat{k} \hat{\ell}+\hat{\ell}^{2}=7, \hat{k}^{2}+2 \hat{k} \hat{\ell}=8, \hat{k}^{2}-\hat{\ell}^{2}=3$, and $\hat{\ell}^{2}+2 \hat{k} \hat{\ell}=5$. The condition (C.6) is not satisfied because $\hat{k}^{2}+\hat{k} \hat{\ell}+\hat{\ell}^{2}=7$ is divisible by $\hat{n}=7$. Stripe patterns, therefore, do not exist.

\section{C.2. Upside-down patterns}

The use of $(a, b, c, d, e, g, h, i, j, s, t, u)=(a, 0,0, d$, $0,0, h, 0,0, s, 0,0)$ in (B.17) and (B.18) leads to $a=1+h+p \hat{n}$ and $d=s+q \hat{n}(p, q \in \mathbb{Z})$. Then $F_{1}$ in $(\mathrm{B} .15)$ is rewritten as

$$
\begin{aligned}
F_{1}\left(z_{1}, 0,0, z_{4}, 0,0, \tilde{\tau}\right) \\
=\sum_{h=0}^{\infty} \sum_{s=0}^{\infty} \sum_{p \in \mathbb{Z}, 1+h+p \hat{n} \geq 0} \sum_{q \in \mathbb{Z}, s+q \hat{n} \geq 0} \\
\quad \times A_{1+h+p \hat{n}, s+q \hat{n}, h s} z_{1}^{1+h+p \hat{n}} z_{4}^{s+q \hat{n}} \bar{z}_{1}^{h} \bar{z}_{4}^{s} \\
\approx A_{1000}^{\prime}(0) \tilde{\tau} z_{1}+A_{2010}\left|z_{1}\right|^{2} z_{1}+A_{1101} z_{1}\left|z_{4}\right|^{2}
\end{aligned}
$$

$\left(A_{a d h s}=A_{a 00 d 00 h 00 s 00}\right)$. We hereafter consider upside-down patterns $\left(z_{1}, \ldots, z_{6}\right)=(w, 0,0, w, 0,0)$ of Type I and $\left(z_{1}, \ldots, z_{6}\right)=(\mathrm{i} w, 0,0, \mathrm{i} w, 0,0)$ of Type II.

For Type I upside-down pattern with $\left(z_{1}, \ldots\right.$, $\left.z_{6}\right)=(w, 0,0, w, 0,0)$, we have

$$
\begin{gathered}
F_{1}(w, 0,0, w, 0,0, \tilde{\tau}) \in \mathbb{R} \\
F_{1}(w, 0,0, w, 0,0, \tilde{\tau}) \approx a_{1} \tilde{\tau} w+a_{6} w^{3} \\
\left(a_{1}=A_{1000}^{\prime}(0) \text { and } a_{6}=A_{2010}(0)+A_{1101}(0)\right) \text { and } \\
F_{4}(w, 0,0, w, 0,0, \tilde{\tau})=F_{1}(w, 0,0, w, 0,0, \tilde{\tau}) \\
F_{2}(w, 0,0, w, 0,0, \tilde{\tau})=F_{5}(w, 0,0, w, 0,0, \tilde{\tau}) \\
=F_{1}(0,0, w, 0, w, 0, \tilde{\tau}) \\
F_{3}(w, 0,0, w, 0,0, \tilde{\tau})=F_{6}(w, 0,0, w, 0,0, \tilde{\tau}) \\
=F_{1}(0, w, 0,0,0, w, \tilde{\tau}) .
\end{gathered}
$$

With the use of $T=\left\{(a, b, \ldots, t, u) \in \mathbb{N}_{+}^{12} \mid\right.$ (B.17), (B.18) $\}, F_{i}=0(i=2,3,5,6)$ in (C.8) are satisfied if

$$
\begin{aligned}
& (0,0, c, 0, e, 0,0,0, j, 0, t, 0) \notin T, \\
& (0, b, 0,0,0, g, 0, i, 0,0,0, u) \notin T .
\end{aligned}
$$

We first consider the case of $(a, b, \ldots, t, u)=(0,0, c$, $0, e, 0,0,0, j, 0, t, 0)$. For this case, (B.17) and (B.18) gives $A \mathbf{x}=\mathbf{b}$ in (C.4 with $\mathbf{x}=(\alpha, \beta, p, q)^{\top}$ $(\alpha, \beta, p, q \in \mathbb{Z}), \alpha=c-j, \beta=e-t$, and

$$
A=\left[\begin{array}{cccc}
-(\hat{k}+\hat{\ell}) & \hat{\ell} & -\hat{n} & 0 \\
\hat{k} & \hat{k} & 0 & -\hat{n}
\end{array}\right] \text {, }
$$

$$
\mathbf{b}=\left[\begin{array}{l}
\hat{k} \\
\hat{\ell}
\end{array}\right] \text {. }
$$


By the condition (C.5), $A \mathbf{x}=\mathbf{b}$ permits an integer solution if

$$
\begin{gathered}
\operatorname{gcd}\left(\hat{k}^{2}+\hat{k} \hat{\ell}+\hat{\ell}^{2}, \hat{k}^{2}+2 \hat{k} \hat{\ell}, \hat{k}^{2}-\hat{\ell}^{2}, \hat{n}\right) \text { is divisible by } \operatorname{gcd}\left(\hat{k}^{2}+2 \hat{k} \hat{\ell}, \hat{n}\right) \\
\Downarrow \\
(\hat{k}-\hat{\ell}) \operatorname{gcd}(\hat{k}, \hat{\ell}) \text { is divisible by } \operatorname{gcd}\left(\hat{k}^{2}+2 \hat{k} \hat{\ell}, \hat{n}\right) .
\end{gathered}
$$

Thus the condition $(0,0, c, 0, e, 0,0,0, j, 0, t, 0) \notin T$ in (C.9) becomes

$$
(\hat{k}-\hat{\ell}) \operatorname{gcd}(\hat{k}, \hat{\ell}) \text { is not divisible by } \operatorname{gcd}\left(\hat{k}^{2}+2 \hat{k} \hat{\ell}, \hat{n}\right) .
$$

We next consider the case of $(a, b, \ldots, t, u)=(0, b, 0,0,0, g, 0, i, 0,0,0, u)$. For this case, (B.17) and (‥18) give $A \mathbf{x}=\mathbf{b}$ in (‥4 $)$ with $\mathbf{x}=(\alpha, \beta, p, q)^{\top}(\alpha, \beta, p, q \in \mathbb{Z}), \alpha=b-i, \beta=g-u$, and

$$
A=\left[\begin{array}{cccc}
\hat{k} & -(\hat{k}+\hat{\ell}) & -\hat{n} & 0 \\
-(\hat{k}+\hat{\ell}) & \hat{\ell} & 0 & -\hat{n}
\end{array}\right], \quad \mathbf{b}=\left[\begin{array}{c}
\hat{k} \\
\hat{\ell}
\end{array}\right] .
$$

By the condition (C.5), $A \mathbf{x}=\mathbf{b}$ permits an integer solution if

$$
\begin{gathered}
\operatorname{gcd}\left(\hat{k}^{2}+\hat{k} \hat{\ell}+\hat{\ell}^{2}, \hat{k}^{2}+2 \hat{k} \hat{\ell}, 2 \hat{k}^{2} \hat{\ell}+\hat{\ell}^{2}, \hat{n}\right) \text { is divisible by } \operatorname{gcd}\left(\hat{k}^{2}+\hat{k} \hat{\ell}+\hat{\ell}^{2}, \hat{n}\right) \\
\Downarrow \\
(\hat{k}-\hat{\ell}) \operatorname{gcd}(\hat{k}, \hat{\ell}) \text { is divisible by } \operatorname{gcd}\left(\hat{k}^{2}+\hat{k} \hat{\ell}+\hat{\ell}^{2}, \hat{n}\right) .
\end{gathered}
$$

Thus the condition $(0, b, 0,0,0, g, 0, i, 0,0,0, u) \notin T$ in C.9 becomes

$$
\begin{array}{r}
(\hat{k}-\hat{\ell}) \operatorname{gcd}(\hat{k}, \hat{\ell}) \text { is not divisible by } \\
\operatorname{gcd}\left(\hat{k}^{2}+\hat{k} \hat{\ell}+\hat{\ell}^{2}, \hat{n}\right) .
\end{array}
$$

To sum up, the upside-down solution of Type I exists if the conditions (C.10) and ([C.11) are satisfied simultaneously.

For Type II upside-down pattern, (C.7) becomes

$$
\begin{aligned}
F_{1}(\mathrm{i} w, & 0,0, \mathrm{i} w, 0,0, \tilde{\tau}) \\
= & \sum_{h=0}^{\infty} \sum_{s=0}^{\infty} \sum_{p \in \mathbb{Z}, 1+h+p \hat{n} \geq 0} \sum_{q \in \mathbb{Z}, s+q \hat{n} \geq 0} \\
& \times A_{1+h+p \hat{n}, s+q \hat{n}, h s}(\mathrm{i} w)^{1+h+p \hat{n}} \\
& \times(\mathrm{i} w)^{s+q \hat{n}}(-\mathrm{i} w)^{h}(-\mathrm{i} w)^{s} \\
= & \mathrm{i} \sum_{h=0}^{\infty} \sum_{s=0}^{\infty} \sum_{p \in \mathbb{Z}, 1+h+p \hat{n} \geq 0} \sum_{q \in \mathbb{Z}, s+q \hat{n} \geq 0} \\
& \times A_{1+h+p \hat{n}, s+q \hat{n}, h s^{1}} \mathrm{i}^{p \hat{n}}(-\mathrm{i})^{q \hat{n}} \\
& \times w^{1+2 h+2 s+(p+q) \hat{n}} .
\end{aligned}
$$

Similarly to the case of stripe patterns (Appendix C.1), Type II upside-down pattern with $\left(z_{1}, \ldots, z_{6}\right)=(\mathrm{i} w, 0,0, \mathrm{i} w, 0,0)$ exists when $\hat{n}$ is even and the conditions (C.10) and (C.11) are satisfied simultaneously.

Example C.2. For $(\hat{k}, \hat{\ell}, \hat{n})=(2,1,7)$, we have

$$
\begin{gathered}
(\hat{k}-\hat{\ell}) \operatorname{gcd}(\hat{k}, \hat{\ell})=(2-1) \operatorname{gcd}(2,1)=2, \\
\operatorname{gcd}\left(\hat{k}^{2}+2 \hat{k} \hat{\ell}, \hat{n}\right)=\operatorname{gcd}(6,7)=1
\end{gathered}
$$

Since $(\hat{k}-\hat{\ell}) \operatorname{gcd}(\hat{k}, \hat{\ell})=2$ is divisible by $\operatorname{gcd}\left(\hat{k}^{2}+\right.$ $2 \hat{k} \hat{\ell}, \hat{n})=1$, the condition (C.11 is not satisfied. Hence the upside-down patterns do not exist.

\section{Appendix D}

\section{Proof of (B.19)}

We would like to obtain the set $S=S_{1} \cup S_{2}$, being defined as

$$
\begin{aligned}
S_{j}= & \left\{(a, b, c, d, e, g, h, i, j, s, t, u) \in \mathbb{N}^{12} \mid\right. \\
& (\text { (B.17) },(\text { (B.18) }), a+b+\cdots+t+u=j\}
\end{aligned}
$$

$(j=1,2)$. It was shown that Ikeda \& Murota, 2014, p. 298]

$$
S_{1}=\{(1,0,0,0,0,0,0,0,0,0,0,0)\} .
$$

In search of the set $S_{2}$, the conditions $1 \leq \ell \leq$ $k-1$ and $2 k+\ell \leq n-1$ are rewritten as

$$
1 \leq \hat{\ell} \leq \hat{k}-1, \quad 0<2 \hat{k}+\hat{\ell} \leq \hat{n}-1
$$


Table D.1. Candidates of $(\alpha, \beta, \gamma)$ for $S_{2}$.

\begin{tabular}{rrrc}
\hline$\alpha$ & $\beta$ & $\gamma$ & $X$ \\
\hline-2 & 0 & 0 & $-3 \hat{k}$ \\
0 & 2 & 0 & $-\hat{k}+2 \hat{\ell}$ \\
0 & 0 & 2 & $-(3 \hat{k}+2 \hat{\ell})$ \\
-1 & 0 & 1 & $-(3 \hat{k}+\hat{\ell})$ \\
0 & -1 & 1 & $-2(\hat{k}+\hat{\ell})$ \\
0 & -1 & -1 & 0 \\
\hline
\end{tabular}

and (B.17) and (B.18) are rewritten as

$$
\left\{\begin{aligned}
X= & \hat{k}(a-h+d-s)-\hat{k}+\hat{\ell}(b-i+e-t) \\
& -(\hat{k}+\hat{\ell})(c-j+g-u) \equiv 0 \bmod \hat{n} \\
Y= & \hat{\ell}(a-h+g-u)-\hat{\ell}+\hat{k}(c-j+e-t) \\
& -(\hat{k}+\hat{\ell})(b-i+d-s) \equiv 0 \bmod \hat{n} .
\end{aligned}\right.
$$

For $S_{2}$, from (D.2), we have $-2 \hat{n}<X<\hat{n}$ and $-2 \hat{n}<Y<\hat{n}$. Then we have

$$
X, Y=0 \text { or }-\hat{n} .
$$

We set

$$
\begin{gathered}
\alpha \equiv a-h+d-s, \quad \beta \equiv b-i+e-t, \\
\gamma \equiv c-j+g-u .
\end{gathered}
$$

Then $X$ in $(\mathrm{D} .3$ reduces to

$$
X=\hat{k}(\alpha-1)+\hat{\ell} \beta-(\hat{k}+\hat{\ell}) \gamma \equiv 0 \bmod \hat{n} .
$$

Table D.2. Noncandidates of $(\alpha, \beta, \gamma)$ for $S_{2}$.

\begin{tabular}{rrrc}
\hline$\alpha$ & $\beta$ & $\gamma$ & $X(\neq 0,-\hat{n})$ \\
\hline 0 & 0 & 0 & $-\hat{k}$ \\
2 & 0 & 0 & $\hat{k}$ \\
0 & -2 & 0 & $-(\hat{k}+2 \hat{\ell})$ \\
0 & 0 & -2 & $\hat{k}+2 \hat{\ell}$ \\
1 & 1 & 0 & $\hat{\ell}$ \\
1 & -1 & 0 & $-\hat{\ell}$ \\
-1 & 1 & 0 & $-2 \hat{k}+\hat{\ell}$ \\
-1 & -1 & 0 & $-(2 \hat{k}+\hat{\ell})$ \\
1 & 0 & 1 & $-(\hat{k}+\hat{\ell})$ \\
1 & 0 & -1 & $\hat{k}+\hat{\ell}$ \\
-1 & 0 & -1 & $-\hat{k}+\hat{\ell}$ \\
0 & 1 & 1 & $-2 \hat{k}$ \\
0 & 1 & -1 & $2 \hat{\ell}$ \\
\hline
\end{tabular}

Table D.3. Candidates of coefficients $(a-u)$ for $S_{2}$.

\begin{tabular}{rrrccc}
\hline \multicolumn{5}{c}{ Nonzero } \\
\multicolumn{1}{c}{$\beta$} & $\gamma$ & Coefficients & $X$ & $Y$ \\
\hline 0 & -1 & -1 & $i=j=1$ & 0 & 0 \\
-1 & 0 & 1 & $c=h=1$ & $-(3 \hat{k}+\hat{\ell})$ & $\hat{k}-2 \hat{\ell}$ \\
0 & 2 & 0 & $b=2$ & $-\hat{k}+2 \hat{\ell}$ & $-(2 \hat{k}+3 \hat{\ell})$ \\
-2 & 0 & 0 & $h=2$ & $-3 \hat{k}$ & $-3 \hat{\ell}$ \\
\hline
\end{tabular}

The value of $X$ is computed for possible values of $(\alpha, \beta, \gamma)$ to classify candidates and noncandidates for $S_{2}$ as listed in Tables D.1 and D.2.

From Table D.1 we hereafter focus on

$$
\begin{aligned}
(\alpha, \beta, \gamma)= & (-2,0,0),(0,2,0),(0,0,2), \\
& (-1,0,1),(0,-1,1),(0,-1,-1)
\end{aligned}
$$

and the value of $Y$ computed is listed in Tables D.3 and D.4 As listed in Table D.3, there are four candidates

\begin{tabular}{|c|c|c|c|c|}
\hline$\alpha$ & $\beta$ & $\gamma$ & Nonzero Coefficients & $Y(\neq 0,-\hat{n})$ \\
\hline-2 & 0 & 0 & $\begin{array}{c}s=2 \\
h=s=1\end{array}$ & $\begin{array}{c}2 \hat{k}+\hat{\ell} \\
\hat{k}-\hat{\ell}\end{array}$ \\
\hline 0 & 2 & 0 & $\begin{array}{c}e=2 \\
b=e=1\end{array}$ & $\begin{array}{c}2 \hat{k}-\hat{\ell} \\
-2 \hat{\ell}\end{array}$ \\
\hline 0 & 0 & 2 & $\begin{array}{c}c=2 \\
g=2 \\
c=g=1\end{array}$ & $\begin{array}{c}2 \hat{k}-\hat{\ell} \\
\hat{\ell} \\
\hat{k}\end{array}$ \\
\hline-1 & 0 & 1 & $\begin{array}{l}h=g=1 \\
s=c=1 \\
s=g=1\end{array}$ & $\begin{array}{c}-\hat{\ell} \\
2 \hat{k} \\
\hat{k}+\hat{\ell}\end{array}$ \\
\hline 0 & -1 & 1 & $\begin{array}{l}i=c=1 \\
i=g=1 \\
t=c=1 \\
t=g=1\end{array}$ & $\begin{array}{c}2 \hat{k} \\
\hat{k}+\hat{\ell} \\
-\hat{\ell} \\
-\hat{k}\end{array}$ \\
\hline 0 & -1 & -1 & $\begin{array}{l}i=u=1 \\
t=j=1 \\
t=u=1\end{array}$ & $\begin{array}{c}\hat{k}-\hat{\ell} \\
-(2 \hat{k}+\hat{\ell}) \\
-(\hat{k}+2 \hat{\ell})\end{array}$ \\
\hline
\end{tabular}

$$
i=j=1, \quad c=h=1, \quad b=2, \quad h=2 .
$$

Among these, $i=j=1$ belongs to $S_{2}$ for any $(\hat{n}, \hat{k}, \hat{\ell}), c=h=1$ and $b=2$ belong to $S_{2}$ only for $(\hat{n}, \hat{k}, \hat{\ell})=(7,2,1)$, and $h=2$ does not belong to $S_{2}$. For $h=2$, we have $Y=-3 \hat{\ell}$. Since we have

Table D.4. Noncandidates of coefficients $(a-u)$ for $S_{2}$. 
$0>Y=-3 \hat{\ell}>-(2 \hat{k}+\hat{\ell})>-\hat{n}, Y=0$ or $-\hat{n}$ in (D.4) cannot be satisfied.

To sum up, for $(\hat{k}, \hat{\ell}, \hat{n})=(2,1,7)$, we have

$$
\begin{aligned}
S_{2}=\{ & (0,0,0,0,0,0,0,1,1,0,0,0), \\
& (0,0,1,0,0,0,1,0,0,0,0,0), \\
& (0,2,0,0,0,0,0,0,0,0,0,0)\}
\end{aligned}
$$

and for other $(\hat{k}, \hat{\ell}, \hat{n})$, we have

$$
S_{2}=\{(0,0,0,0,0,0,0,1,1,0,0,0)\} .
$$

The combination of these with (D.1) gives $S$. This suffices to prove (B.19). 